\title{
BICOMPLEX SIGNALS WITH SPARSITY CONSTRAINTS
}

\author{
P. CEREJEIRAS ${ }^{\dagger}$, Y. FU ${ }^{\ddagger}$, AND N. GOMES*
}

\begin{abstract}
In this paper we aim to proof the possibility to reconstruct a bicomplex sparse signal, with high probability, from a reduced number of bicomplex random samples. Due to the idempotent representation of the bicomplex algebra this case is similar to the case of the standard Fourier basis, thus allowing us to adapt in a rather easy way the arguments from the recent works of Rauhut and Candés et al..
\end{abstract}

Keywords: Compressed Sensing; Sparsity; Bicomplex Signals;

AMS classification: Primary: 30G35; Secondary: 26A33, 30A05, 31B05;

\section{INTRODUCTION}

An important problem in signal processing is the possibility of reconstruction of a given signal from a few of its samples. This problem is closely related to nonlinear and adaptive approximation. Indeed, classic linear schemes in general do not apply here. One way to overcome this difficulty is to search for the atom which has a locally optimal fit at each step - the so called greedy algorithm. However, this procedure has exponentially growing computational costs and does not necessarily lead to exact (or even to optimal) reconstructions. In the last decades several approaches were developed to overcome this shortness. One such approach is Compressed Sensing (CS) [6, 3]. The main idea behind CS is that under certain conditions on the sampling matrix one can obtain a sparse reconstruction of the signal, i.e., if we have an a-priori knowledge that the representation of the signal in a given dictionary has only a few non-zero coefficients then we can reconstruct it from those few samples by means of a simple basis pursuit procedure. The condition on the sampling matrix, or RIP condition, states that the matrix behaves almost like an isometry. Now, this also represents a problem since in practice this is almost never the case (e.g. [15]). However, and as suggested by Rauhut in [14], this requirement can be lowered to be fulfilled with a certain (high) probability. This is a more realistic scenario since in many application the worst-cases are seldom or can entirely be avoided. Hereby, we propose to follow the idea of the reconstruction of the signal by basis pursuit via a $\ell_{1}$-minimization procedure $[2,4,5]$.

In this paper we are interested in the case of bicomplex signals depending on two variables. The motivation comes from the fact that higher-dimensional algebraic structures can be used to represent color image encoding in image processing which has attracted a lot of attention during the last decade. In [12] the authors point out that the so-called IHS-color spaces representation (i.e., Intensity-Hue-Saturation) which has broad applications, particularly in human vision, can be mathematically represented by having values in bicomplex numbers. This means that a color image based on this coding scheme can be represented by a function depending on two variables and taking 
values in the bicomplex numbers. Another possible application of these kind of bicomplex signals can be found in multi-channel information [11] which can easily be adapted to our setting.

The paper is organized as follows. In Section 2. we introduce the necessary tools. In Section 3. we present the setting of our problem, the main theorems and prove some auxiliary lemmas. Finally, Section 4. is dedicated to the detailed proof of the main theorems.

\section{Preliminaries}

As a realization of a commutative $4 D$ real algebra, which is not a division algebra, we have the commutative ring of bicomplex numbers, denoted as $\mathbb{B} \mathbb{C}$. Here, $\mathbf{i}$ and $\mathbf{j}$ are commuting imaginary units, i.e.,

$$
\mathbf{i j}=\mathbf{j i}=\mathbf{k}, \quad \mathbf{i}^{2}=\mathbf{j}^{2}=-1, \text { whereas } \mathbf{k}^{2}=+1,
$$

and

$$
\mathbb{B} \mathbb{C}:=\{\zeta=a+b \mathbf{i}+c \mathbf{j}+d \mathbf{k}, a, b, c, d \in \mathbb{R}\} .
$$

Based on identities (2.1) we decompose $\mathbb{B} \mathbb{C}$ into two ways. Indeed, $\mathbb{B C}=\mathbb{C}(\mathbf{i})+\mathbf{j} \mathbb{C}(\mathbf{i})$, that is

$$
\zeta=a+b \mathbf{i}+c \mathbf{j}+d \mathbf{k}=z_{1}+z_{2} \mathbf{j}, \quad \text { with } z_{1}=a+b \mathbf{i}, z_{2}=c+d \mathbf{i} \in \mathbb{C}(\mathbf{i}),
$$

where $\mathbb{C}(\mathbf{i})$ is the set of complex numbers with the imaginary unit $\mathbf{i}$. We introduce the automorphism, or conjugation, given by

$$
\zeta:=z_{1}+z_{2} \mathbf{j} \mapsto \bar{\zeta}:=\bar{z}_{1}-\bar{z}_{2} \mathbf{j},
$$

where $\bar{z}_{j}, j=1,2$, denotes the usual complex conjugation in $\mathbb{C}(\mathbf{i})$.

Likewisely, we have $\mathbb{B C}=\mathbb{C}(\mathbf{j})+\mathbf{i} \mathbb{C}(\mathbf{j})$. Also the set of hyperbolic numbers $\mathbb{D}:=\{a+d \mathbf{k}: a, d \in \mathbb{R}\}$ can be embed into $\mathbb{B} \mathbb{C}$ as follows: $z=a+d \mathbf{k} \sim a+0 \mathbf{i}+0 \mathbf{j}+d \mathbf{i j} \in \mathbb{B} \mathbb{C}$.

Every bicomplex number $\zeta=z_{1}+z_{2} \mathbf{j}$ satisfying to $z_{1}^{2}+z_{2}^{2} \neq 0$ is invertible, and its inverse is given by $\zeta^{-1}=\frac{z_{1}-z_{2} \mathbf{j}}{z_{1}^{2}+z_{2}^{2}}$. If, on the other hand, $z_{1}^{2}+z_{2}^{2}=0$ then $\zeta$ is a zero divisor in $\mathbb{B} \mathbb{C}$. In fact, there are no other zero divisors in $\mathbb{B C}$. We turn our attention to two special zero divisors. We define

$$
\mathbf{e}^{+}=\frac{1}{2}(1+\mathbf{i j}), \quad \mathbf{e}^{-}=\frac{1}{2}(1-\mathbf{i j}) .
$$

Straightforward computations show that they are zero divisors as well as mutually complementary idempotent elements

$$
\mathbf{e}^{+} \mathbf{e}^{-}=\mathbf{e}^{-} \mathbf{e}^{+}=0, \quad \mathbf{e}^{+}+\mathbf{e}^{-}=1, \quad\left(\mathbf{e}^{+}\right)^{2}=\mathbf{e}^{+}, \quad\left(\mathbf{e}^{-}\right)^{2}=\mathbf{e}^{-} .
$$

Thus, the two sets $\mathbb{B C}_{\mathbf{e}^{+}}:=\mathbb{B} \mathbb{C e}^{+}$and $\mathbb{B C}_{\mathbf{e}^{-}}:=\mathbb{B C} \mathbf{e}^{-}$are principal ideals in the ring $\mathbb{B C}$. Given an open set $X \subset \mathbb{B C}$, we shall denote its projections $\Pi_{\mathbf{e}^{ \pm}}$into the principal ideals $\mathbb{B C}_{\mathbf{e}^{ \pm}}$, resp., as $X_{1}=\Pi_{\mathbf{e}^{+}} X \subset \mathbb{B C}_{\mathbf{e}^{+}}, \quad X_{2}=\Pi_{\mathbf{e}^{-}} X \subset \mathbb{B C}_{\mathbf{e}^{-}}$. Taking this decomposition into account we have $X=X_{1} \mathbf{e}^{+}+X_{2} \mathbf{e}^{-}$, with $X_{1} \mathbf{e}^{+}=X \mathbf{e}^{+}$and $X_{2} \mathbf{e}^{-}=X \mathbf{e}^{-}$. This leads to the following representation

$$
\zeta=z_{1}+z_{2} \mathbf{j}=\beta_{1} \mathbf{e}^{+}+\beta_{2} \mathbf{e}^{-}, \quad \text { where } \beta_{1}=z_{1}-\mathbf{i} z_{2}, \beta_{2}=z_{1}+\mathbf{i} z_{2} \in \mathbb{C}(\mathbf{i}) .
$$


Moreover, if both $\beta_{1}, \beta_{2}$ are invertible in $\mathbb{C}(\mathbf{i})$ then $\zeta$ is invertible, and we have

$$
\zeta^{-1}=\beta_{1}^{-1} \mathbf{e}^{+}+\beta_{2}^{-1} \mathbf{e}^{-}=\frac{\overline{\beta_{1}}}{\left|\beta_{1}\right|^{2}} \mathbf{e}^{+}+\frac{\overline{\beta_{2}}}{\left|\beta_{2}\right|^{2}} \mathbf{e}^{-} .
$$

Based on representation (2.7) we have the so-called hyperbolic norm (see [1])

$$
|\zeta|_{\mathbf{k}}=\left|\beta_{1}\right| \mathbf{e}^{+}+\left|\beta_{2}\right| \mathbf{e}^{-} .
$$

Let us point out that although (2.8) is not a norm strictly speaking its real part induces a norm $|\cdot|: \mathbb{B C} \rightarrow \mathbb{R}_{0}^{+}$given by

$$
|\zeta|:=\operatorname{Re}\left(|\zeta|_{\mathbf{k}}\right)=\operatorname{Re}\left(\left|\beta_{1}\right| \mathbf{e}^{+}+\left|\beta_{2}\right| \mathbf{e}^{-}\right):=\frac{1}{2}\left(\left|\beta_{1}\right|+\left|\beta_{2}\right|\right) .
$$

We now look into idempotent representations of matrices taking values in $\mathbb{B} \mathbb{C}$. We denote by $\mathbb{B C}^{m \times n}$ the set of $m \times n$ matrices with bicomplex entries. As in the scalar case, the operations over the matrices can be realized component-wise keeping in mind the non-commutativity of matrix multiplication. Note that $\mathbb{B} \mathbb{C}^{m \times n}$ is not a vectorial space, but a $\mathbb{B} \mathbb{C}$-module.

Given such a matrix $A=\left(a_{i, j}\right) \in \mathbb{B} \mathbb{C}^{m \times n}$ its idempotent representation is obtained by accordingly decomposing each of its entries by $(2.7)$, that is,

$$
A=[A]_{1} \mathbf{e}^{+}+[A]_{2} \mathbf{e}^{-},
$$

where $[A]_{1}=\Pi_{\mathbf{e}^{+}} A,[A]_{2}=\Pi_{\mathbf{e}^{-}} A$ are now $m \times n$ matrices taking values in $\mathbb{C}(\mathbf{i})$. In consequence, we shall write $[A]_{1},[A]_{2} \in \mathbb{C}^{m \times n}(\mathbf{i})$, and we have $\mathbb{B} \mathbb{C}^{m \times n}=\mathbb{C}^{m \times n}(\mathbf{i}) \mathbf{e}^{+}+\mathbb{C}^{m \times n}(\mathbf{i}) \mathbf{e}^{-}$.

Definition 2.1. Given a square matrix $A=[A]_{1} \mathbf{e}^{+}+[A]_{2} \mathbf{e}^{-} \in \mathbb{C}^{n \times n}(\mathbf{i}) \mathbf{e}^{+}+\mathbb{C}^{n \times n}(\mathbf{i}) \mathbf{e}^{-}=\mathbb{B} \mathbb{C}^{n \times n}$, we define its determinant $\operatorname{det} A$ by $\operatorname{det} A:=\left(\operatorname{det}[A]_{1}\right) \mathbf{e}^{+}+\left(\operatorname{det}[A]_{2}\right) \mathbf{e}^{-}$.

Lemma 2.1 ([1], Corollary 2.2.2). Let be $A$ and $B$ be two square bicomplex matrices. Then $\operatorname{det}(A B)=\operatorname{det} A \operatorname{det} B$.

Lemma 2.2 ([1], Proposition 2.2.3). A square bicomplex matrix $A=[A]_{1} \mathbf{e}^{+}+[A]_{2} \mathbf{e}^{-} \in \mathbb{C}^{n \times n}(\mathbf{i}) \mathbf{e}^{+}+$ $\mathbb{C}^{n \times n}(\mathbf{i}) \mathbf{e}^{-}=\mathbb{B C}^{n \times n}$ is invertible if and only if $[A]_{1},[A]_{2}$ are invertible in $\mathbb{C}^{n \times n}(\mathbf{i})$.

\section{Sparse sampling of Bicomplex signals}

We now consider the problem of reconstructing a sparse bicomplex signal. We shall begin with a description of our setting, after which we present our main results.

3.1. The Bicomplex Setting. We consider bicomplex trigonometric waves of type

$$
\zeta_{k, \tilde{k}}(x, y)=e^{\mathbf{i} k x} \mathbf{e}^{+}+e^{\mathbf{i} \tilde{k} y} \mathbf{e}^{-}, \quad x, y \in[0,2 \pi],
$$

with $k, \tilde{k} \in \mathbb{Z}$. As in [14], we denote by $\prod_{\rho}$ the space of all bicomplex trigonometric polynomials of maximal order $\rho \in \mathbb{N}_{0}$ in two real variables. For convenience, we use $\mathbb{Z}_{\rho}^{2}=[-\rho, \rho]^{2} \cap \mathbb{Z}^{2}$. Thus, an element $f \in \prod_{\rho}$ is of the form

$$
f(x, y)=\sum_{(k, \tilde{k}) \in \mathbb{Z}_{\rho}^{2}} c_{k, \tilde{k}}\left(e^{\mathbf{i} k x} \mathbf{e}^{+}+e^{\mathbf{i} \tilde{k} y} \mathbf{e}^{-}\right)=\sum_{(k, \tilde{k}) \in \mathbb{Z}_{\rho}^{2}}\left(c_{k} e^{\mathbf{i} k x} \mathbf{e}^{+}+c_{\tilde{k}} e^{\mathbf{i} \tilde{k} y} \mathbf{e}^{-}\right),
$$


with $(x, y) \in[0,2 \pi]^{2}$, and some sequence of coefficients $c=\left(c_{k, \tilde{k}}\right)_{k, \tilde{k}}$, where

$$
c_{k, \tilde{k}}=c_{k} \mathbf{e}^{+}+c_{\tilde{k}} \mathbf{e}^{-} \in \mathbb{C}=\mathbb{C}(\mathbf{i}) \mathbf{e}^{+}+\mathbb{C}(\mathbf{i}) \mathbf{e}^{-} .
$$

Furthermore, we assume that the sequence $c$ of coefficients has support on a set $T \subset \mathbb{Z}_{\rho}^{2}$ which is much smaller than the dimension $D=(2 \rho+1)^{2}$ of $\prod_{\rho}$. In other words, the finite combination in (3.2) is sparse, that is, only a few coefficients $c_{k, \tilde{k}}$ are non-zero.

As we do not possess any information on $T$ except its maximum size, we introduce the auxiliary (non linear) space $\prod_{\rho}(M)$ of all polynomials of type (3.2) such that the sequence of coefficients $c$ has support on a set $T \subset \mathbb{Z}_{\rho}^{2}$ satisfying to $|T| \leq M$, i.e., $f \in \prod_{\rho}(M) \subset \prod_{\rho}$ is of the form

$$
f(x, y)=\sum_{(k, \tilde{k}) \in T,|T| \leq M} c_{k, \tilde{k}}\left(e^{\mathbf{i} k x} \mathbf{e}^{+}+e^{\mathbf{i} \tilde{k} y} \mathbf{e}^{-}\right), \quad(x, y) \in[0,2 \pi]^{2},
$$

and we say $f$ has sparsity $M$.

We now state our problem: given a sampling set of $N$ independent random variables having uniform distribution on $[0,2 \pi]^{2}$, and assuming the signal $f$ to be sparse, that is $f \in \prod_{\rho}(M),(M \ll$ $D)$, we wish to reconstruct $f$ from its known samples at the chosen sampling set.

3.2. Main theorems. As indicated before, we avoid the usage of basis pursuit methods as they are in general too restrictive and computationally expensive for most applications. Instead, we provide a probabilistic answer to our problem. The theorems below are analogues to the ones in Candés, Romberg, and Tao [3] and in Rauhut [14].

First, we state the following $\ell_{1}$-minimization problem: given a set of samples $\left\{f\left(x_{j}, y_{j}\right), j=\right.$ $1, \ldots, N\}$ we wish to find a sequence $c=\left(c_{k, \tilde{k}}\right) \in \ell_{2}\left(\mathbb{Z}_{\rho}^{2}\right)$ solving

$$
\min \|c\|_{\ell_{1}} \quad \text { s.t. } \quad g\left(x_{j}, y_{j}\right):=\sum_{(k, \tilde{k}) \in \mathbb{Z}_{\rho}^{2}} c_{k, \tilde{k}}\left(e^{\mathbf{i} k x_{j}} \mathbf{e}^{+}+e^{\mathbf{i} \tilde{k} y_{j}} \mathbf{e}^{-}\right)=f\left(x_{j}, y_{j}\right),
$$

for all $j=1, \ldots, N$, and where

$$
\|c\|_{\ell_{1}}:=\sum_{(k, \tilde{k}) \in \mathbb{Z}_{\rho}^{2}} 2 \operatorname{Re}\left(\left|c_{k, \tilde{k}}\right|_{\mathbf{k}}\right)=\sum_{(k, \tilde{k}) \in \mathbb{Z}_{\rho}^{2}}\left(\left|c_{k}\right|+\left|c_{\tilde{k}}\right|\right) .
$$

The following theorems state when the signal $f$ can be reconstructed by means of solving this minimization problem.

Theorem 3.1. Assume $f \in \prod_{\rho}(M)$ and let $X=\left\{\left(x_{1}, y_{1}\right), \ldots,\left(x_{N}, y_{N}\right)\right\} \subset[0,2 \pi]^{2}$ be a set of independent random variables having uniform distribution on $[0,2 \pi]^{2}$.

Choose $n \in \mathbb{N}, \beta>0, \kappa>0$ and $K_{1}, \cdots, K_{n} \in \mathbb{N}$ such that

$$
a:=\sum_{m=1}^{n} \beta^{n / K_{m}}<1 \text { and } \frac{\kappa}{1-\kappa} \leq \frac{1-a}{1+a} M^{-3 / 2} .
$$


Set $\theta:=N / M$. Then, $f$ can be reconstructed exactly from its sample values $f\left(x_{1}, y_{1}\right), \ldots, f\left(x_{N}, y_{N}\right)$ by solving the $\ell_{1}$-minimization problem (3.5) with a probability of, at least,

$$
1-\left(D \beta^{-2 n} \sum_{m=1}^{n} G_{2 m K_{m}}(\theta)+\kappa^{-2} M G_{2 n}(\theta)\right),
$$

where, we recall, $D=(2 \rho+1)^{2}$ and $G_{2 s}(\theta):=\theta^{-2 s} \sum_{k=1}^{s} S_{2}(2 s, k) \theta^{k}$ for $s \in \mathbb{N}$, and where $S_{2}(\cdot, \cdot)$ denotes the Stirling numbers of second order.

While the above theorem provides exact constants we can give a version of the theorem which is somewhat easier to apply.

Theorem 3.2. Assume $f \in \prod_{\rho}(M)$ and let $X=\left\{\left(x_{1}, y_{1}\right), \ldots,\left(x_{N}, y_{N}\right)\right\} \subset[0,2 \pi]^{2}$ be a set of independent random variables having uniform distribution on $[0,2 \pi]^{2}$.

If for some $\epsilon>0$, there exists a constant $C>0$ such that it holds

$$
N \geq C M \log (D / \epsilon)
$$

then, with a probability at least $1-\epsilon$, the signal $f$ can be recovered from its sample values $f\left(x_{1}, y_{1}\right), \ldots, f\left(x_{N}, y_{N}\right)$ by solving the minimization problem (3.5).

The proof of these two theorems requires several additional lemmas. These will be presented and proved in the next subsection. Although some parts follow closely Rauhut [14], we have emphasize the need to adapt many of the tools to our own bicomplex case, since the algebraic construction is quite different.

3.3. Additional Lemmas. We begin by introducing the spaces of functions: let $\ell_{2}\left(\mathbb{Z}_{\rho}^{2}\right), \ell_{2}(T)$, and $\ell_{2}(X)$, denote the $\ell_{2}$-spaces w.r.t. the norm (2.9) of sequences indexed by the grids $\mathbb{Z}_{\rho}^{2}, T$, and $X$, respectively.

Secondly, we consider the sampling operator $\mathcal{F}_{X}: \ell_{2}\left(\mathbb{Z}_{\rho}^{2}\right) \rightarrow \ell_{2}(X)$ given by

$$
\mathcal{F}_{X}(c)\left(x_{j}, y_{j}\right):=\sum_{(k, \tilde{k}) \in \mathbb{Z}_{\rho}^{2}} c_{k, \tilde{k}}\left(e^{\mathbf{i} k x_{j}} \mathbf{e}^{+}+e^{\mathbf{i} \tilde{k} y_{j}} \mathbf{e}^{-}\right)_{j=1, \ldots, N},
$$

as well as its restriction, $\mathcal{F}_{T X}$, to sequences with support on $T$. Thus, $\mathcal{F}_{T X}$ is an operator acting from $\ell_{2}(T)$ in $\ell_{2}(X)$. Also, we consider their adjoint operators, $\mathcal{F}_{X}^{*}: \ell_{2}(X) \rightarrow \ell_{2}\left(\mathbb{Z}_{\rho}^{2}\right)$ and $\mathcal{F}_{T X}^{*}:$ $\ell_{2}(X) \rightarrow \ell_{2}(T)$.

Finally, as an abuse of language, and when clear from the context, we shall denote the sample sequence by the same letter of the original signal, that is to say, $f=\left(f\left(x_{j}, y_{j}\right)\right)_{j=1, \ldots, N} \in \ell_{2}(X)$.

So we reformulate our problem: we want to reconstruct a sequence $c \in \ell_{2}\left(\mathbb{Z}_{\rho}^{2}\right)$ from the data $\mathcal{F}_{X} c=f$, where $f$ is the sample sequence of the signal in $\ell_{2}(X)$, by solving the following $\ell_{1^{-}}$ minimization problem

$$
\min \|c\|_{\ell_{1}} \quad \text { subject to } \quad \mathcal{F}_{X} c=f \text {. }
$$


We start by define the analogue to sign function in the bicomplex case as

$$
\operatorname{sgn}(\zeta)=\operatorname{sgn}\left(\beta_{1} \mathbf{e}^{+}+\beta_{2} \mathbf{e}^{-}\right):=\operatorname{sgn}\left(\beta_{1}\right) \mathbf{e}^{+}+\operatorname{sgn}\left(\beta_{2}\right) \mathbf{e}^{-},
$$

where $\operatorname{sgn} \beta$, for $\beta \in \mathbb{C}(\mathbf{i})$, is the complex sign function $\operatorname{sgn} \beta=\frac{\beta}{|\beta|}$, if $\beta \neq 0$, and 0 otherwise. For an arbitrary sequence of coefficients $c:=\left(c_{k, \tilde{k}}\right)_{k, \tilde{k}}=\left(c_{k} \mathbf{e}^{+}+c_{\tilde{k}} \mathbf{e}^{-}\right)_{k, \tilde{k}}$ then $\operatorname{sgn} c_{k, \tilde{k}}=0$ if $(k, \tilde{k}) \notin \operatorname{supp} c$, while $\left|\operatorname{sgn} c_{k, \tilde{k}}\right|=1$ for all $(k, \tilde{k}) \in \operatorname{supp} c$ where the norm $|\cdot|$ is given by $(2.9)$.

Lemma 3.1. Given two bicomplex numbers $c=c_{1} \mathbf{e}^{+}+c_{2} \mathbf{e}^{-}$and $g=g_{1} \mathbf{e}^{+}+g_{2} \mathbf{e}^{-}$, such that both $c_{1}, c_{2}$ are non-zero, then it holds

(1) $|\operatorname{sgn} c|_{\mathbf{k}}=1$;

(2) $c \overline{\operatorname{sgn} c}=|c|_{\mathbf{k}}$;

(3) $|g|_{\mathbf{k}}|\operatorname{sgn} c|_{\mathbf{k}}=|g \operatorname{sgn} c|_{\mathbf{k}}$.

Proof. By (3.10) we have $\operatorname{sgn} c=\left(\operatorname{sgn} c_{1}\right) \mathbf{e}^{+}+\left(\operatorname{sgn} c_{2}\right) \mathbf{e}^{-}$, so that by $(2.8)$ we get $|\operatorname{sgn} c|_{\mathbf{k}}=\left|\operatorname{sgn} c_{1}\right| \mathbf{e}^{+}+$ $\left|\operatorname{sgn} c_{2}\right| \mathbf{e}^{-}$. Hence, when both $c_{1}, c_{2}$ are non-zero we obtain

$$
|\operatorname{sgn} c|_{\mathbf{k}}=\left|\operatorname{sgn} c_{1}\right| \mathbf{e}^{+}+\left|\operatorname{sgn} c_{2}\right| \mathbf{e}^{-}=\left|\frac{c_{1}}{\left|c_{1}\right|}\right| \mathbf{e}^{+}+\left|\frac{c_{2}}{\left|c_{2}\right|}\right| \mathbf{e}^{-}=\mathbf{e}^{+}+\mathbf{e}^{-}=1,
$$

which proves (1).

For (2) we observe that $\overline{\mathbf{e}^{ \pm}}=\mathbf{e}^{ \pm}$and so

$$
\begin{gathered}
c \overline{\operatorname{sgn} c}=\left(c_{1} \mathbf{e}^{+}+c_{2} \mathbf{e}^{-}\right)\left(\frac{\overline{c_{1}}}{\left|c_{1}\right|} \mathbf{e}^{+}+\frac{\overline{c_{2}}}{\left|c_{2}\right|} \mathbf{e}^{-}\right)=\left(\frac{c_{1} \overline{c_{1}}}{\left|c_{1}\right|} \mathbf{e}^{+}+\frac{c_{2} \overline{c_{2}}}{\left|c_{2}\right|} \mathbf{e}^{-}\right) \\
=\left|c_{1}\right| \mathbf{e}^{+}+\left|c_{2}\right| \mathbf{e}^{-}=|c|_{\mathbf{k}} .
\end{gathered}
$$

Finally, for (3) we have for the left hand side,

$$
|g|_{\mathbf{k}}|\operatorname{sgn} c|_{\mathbf{k}}=|g|_{\mathbf{k}}
$$

due to (1), while for the right hand side,

$$
\begin{aligned}
|g \operatorname{sgn} c|_{\mathbf{k}}= & \left|\left(g_{1} \mathbf{e}^{+}+g_{2} \mathbf{e}^{-}\right)\left(\frac{c_{1}}{\left|c_{1}\right|} \mathbf{e}^{+}+\frac{c_{2}}{\left|c_{2}\right|} \mathbf{e}^{-}\right)\right|_{\mathbf{k}}=\left|\frac{g_{1} c_{1}}{\left|c_{1}\right|} \mathbf{e}^{+}+\frac{g_{2} c_{2}}{\left|c_{2}\right|} \mathbf{e}^{-}\right|_{\mathbf{k}} \\
& =\left|\frac{g_{1} c_{1}}{\left|c_{1}\right|}\right| \mathbf{e}^{+}+\left|\frac{g_{2} c_{2}}{\left|c_{2}\right|}\right| \mathbf{e}^{-}=\left|g_{1}\right| \mathbf{e}^{+}+\left|g_{2}\right| \mathbf{e}^{-}=|g|_{\mathbf{k}} .
\end{aligned}
$$

The following lemma is an adaptation of [3], Lemma 2.1, to our setting.

Lemma 3.2. Let $c=\left(c_{k, \tilde{k}}\right) \in \ell_{2}\left(\mathbb{Z}_{\rho}^{2}\right)$ be a sequence with support on $T$ and satisfying to $\mathcal{F}_{T X} c=f$. Also, assume $\mathcal{F}_{T X}: \ell_{2}(T) \rightarrow \ell_{2}(X)$ to be injective. If there exists a sequence $P \in \ell_{2}\left(\mathbb{Z}_{\rho}^{2}\right)$ with the following properties:

(1) $\left|P_{k \tilde{k}}\right|=1$ for all $(k, \tilde{k}) \in T$;

(2) $\left|P_{k \tilde{k}}\right|<1$ for all $(k, \tilde{k}) \notin T$;

(3) there exists a $\lambda=\lambda_{1} \mathbf{e}^{+}+\lambda_{2} \mathbf{e}^{-} \in \ell_{2}(X)$ such that $P=\left[\mathcal{F}_{X}^{*}\right]_{1} \lambda_{1} \mathbf{e}^{+}+\left[\mathcal{F}_{X}^{*}\right]_{2} \lambda_{2} \mathbf{e}^{-}$, then $c$ is the unique minimizer to problem (3.9). 
Proof. First, we recall that $|\cdot|:=\operatorname{Re}\left(|\cdot|_{\mathbf{k}}\right)$ denotes the norm $(2.9)$ while $\left[\mathcal{F}_{X}^{*}\right]_{1},\left[\mathcal{F}_{X}^{*}\right]_{2}$ denote the components of the idempotent representation $\mathcal{F}_{X}^{*}=\left[\mathcal{F}_{X}^{*}\right]_{1} \mathbf{e}^{+}+\left[\mathcal{F}_{X}^{*}\right]_{2} \mathbf{e}^{-}$.

The case where $T$ is empty is trivial. The same happens if $f$ is zero everywhere on $X$. Therefore, we assume $f=\left(f\left(x_{j}, y_{j}\right), j=1, \ldots, N\right)$ to be a non-zero vector.

Let $h=\left(h_{k, \tilde{k}}\right) \in \ell_{2}\left(\mathbb{Z}_{\rho}^{2}\right)$ be an arbitrary vector such that $\mathcal{F}_{X} h=\mathcal{F}_{X} c$. Take $g=h-c$. Then, $\mathcal{F}_{X} g=0$ on $X$ and $\left|h_{k, \tilde{k}}\right|_{\mathbf{k}}=\left|c_{k, \tilde{k}}+g_{k, \tilde{k}}\right|_{\mathbf{k}}$ for all $(k, \tilde{k}) \in T$.

First, we assume $(k, \tilde{k}) \in T$ such that both $c_{k}, c_{\tilde{k}}$ are non-zero. Then,

$$
\overline{\operatorname{sgn} c_{k, \tilde{k}}} \operatorname{sgn} c_{k, \tilde{k}}=\left(\frac{c_{k}}{\left|c_{k}\right|} \mathbf{e}^{+}+\frac{c_{\tilde{k}}}{\left|c_{\tilde{k}}\right|} \mathbf{e}^{-}\right)\left(\frac{\bar{c}_{k}}{\left|c_{k}\right|} \mathbf{e}^{+}+\frac{\bar{c}_{\tilde{k}}}{\left|c_{\tilde{k}}\right|} \mathbf{e}^{-}\right)=\mathbf{e}^{+}+\mathbf{e}^{-}=1,
$$

so that

$$
\begin{aligned}
\left|h_{k, \tilde{k}}\right|_{\mathbf{k}} & =\left|\left(c_{k, \tilde{k}}+g_{k, \tilde{k}}\right) \overline{\operatorname{sgn} c_{k, \tilde{k}}} \operatorname{sgn} c_{k, \tilde{k}}\right|_{\mathbf{k}} \\
& =\left|\left(c_{k, \tilde{k}} \overline{\operatorname{sgn} c_{k, \tilde{k}}}+g_{k, \tilde{k}} \overline{\operatorname{sgn} c_{k, \tilde{k}}}\right) \operatorname{sgn} c_{k, \tilde{k}}\right|_{\mathbf{k}} \\
& =\left.|| c_{k, \tilde{k}}\right|_{\mathbf{k}}+\left.g_{k, \tilde{k}} \overline{\operatorname{sgn} c_{k, \tilde{k}}}\right|_{\mathbf{k}}\left|\overline{\operatorname{sgn} c_{k, \tilde{k}}}\right|_{\mathbf{k}} \quad \text { Lemma 3.1, (2) and (3) } \\
& =\left.|| c_{k, \tilde{k}}\right|_{\mathbf{k}}+\left.g_{k, \tilde{k}} \overline{\operatorname{sgn} c_{k, \tilde{k}}}\right|_{\mathbf{k}},
\end{aligned}
$$

We apply the idempotent decomposition (2.7) to each sequence, namely,

$$
c_{k, \tilde{k}}=c_{k} \mathbf{e}^{+}+c_{\tilde{k}} \mathbf{e}^{-}, \quad g_{k, \tilde{k}}=g_{k} \mathbf{e}^{+}+g_{\tilde{k}} \mathbf{e}^{-}, \quad h_{k, \tilde{k}}=h_{k} \mathbf{e}^{+}+h_{\tilde{k}} \mathbf{e}^{-} .
$$

Since the norm (2.9) is given by $\left|z_{1} \mathbf{e}^{+}+z_{2} \mathbf{e}^{-}\right|:=\operatorname{Re}\left(\left|z_{1} \mathbf{e}^{+}+z_{2} \mathbf{e}^{-}\right|_{\mathbf{k}}\right)=\frac{1}{2}\left(\left|z_{1}\right|+\left|z_{2}\right|\right)$, for all $z_{1}, z_{2} \in \mathbb{C}(\mathbf{i})$, expression (3.11) can be further simplified as

$$
\begin{aligned}
2 \operatorname{Re}\left(\left|h_{k, \tilde{k}}\right|_{\mathbf{k}}\right) & =2 \operatorname{Re}\left(\left.|| c_{k, \tilde{k}}\right|_{\mathbf{k}}+\left.g_{k, \tilde{k}} \overline{\operatorname{sgn} c_{k, \tilde{k}}}\right|_{\mathbf{k}}\right) \\
& =2 \operatorname{Re}\left(|| c_{k}\left|\mathbf{e}^{+}+\right| c_{\tilde{k}}\left|\mathbf{e}^{-}+g_{k} \overline{\operatorname{sgn} c_{k}} \mathbf{e}^{+}+g_{\tilde{k}} \overline{\operatorname{sgn} c_{\tilde{k}}} \mathbf{e}^{-}\right|_{\mathbf{k}}\right) \\
& =|| c_{k}\left|+g_{k} \overline{\operatorname{sgn} c_{k}}\right|+|| c_{\tilde{k}}\left|+g_{\tilde{k}} \overline{\operatorname{sgn} c_{\tilde{k}}}\right| .
\end{aligned}
$$

Moreover,

$$
\begin{aligned}
|| c_{k}\left|+g_{k} \overline{\operatorname{sgn} c_{k}}\right|+|| c_{\tilde{k}}\left|+g_{\tilde{k}} \overline{\operatorname{sgn} c_{\tilde{k}}}\right| & \geq\left|c_{k}\right|+\operatorname{Re}\left(g_{k} \overline{\operatorname{sgn} c_{k}}\right)+\left|c_{\tilde{k}}\right|+\operatorname{Re}\left(g_{\tilde{k}} \overline{\operatorname{sgn} c_{\tilde{k}}}\right) \\
& =\left|c_{k}\right|+\left|c_{\tilde{k}}\right|+2 \operatorname{Re}\left(g_{k} \overline{\operatorname{sgn} c_{k}} \mathbf{e}^{+}+g_{\tilde{k}} \overline{\operatorname{sgn} c_{\tilde{k}}} \mathbf{e}^{-}\right) \\
& =2\left|c_{k, \tilde{k}}\right|+2 \operatorname{Re}\left(g_{k, \tilde{k}} \overline{\operatorname{sgn} c_{k, \tilde{k}}}\right) .
\end{aligned}
$$

Now, we consider the case where $(k, \tilde{k}) \in T$ and one (and only one) of the coefficients $c_{k}, c_{\tilde{k}}$ is zero. Without lost of generality, we assume $c_{k} \neq 0$ and $c_{\tilde{k}}=0$. In this case we have

$$
\left|h_{k, \tilde{k}}\right|_{\mathbf{k}}=\left|c_{k, \tilde{k}}+g_{k, \tilde{k}}\right|_{\mathbf{k}}=\left|c_{k} \mathbf{e}^{+}+g_{k, \tilde{k}}\right|_{\mathbf{k}} .
$$

We introduce the auxiliar element $d_{k, \tilde{k}}:=c_{k} \mathbf{e}^{+}+c_{k} \mathbf{e}^{-}$(that is to say, we replace the $\tilde{k}$-coefficient by $c_{k}$ ). Then,

$$
\begin{aligned}
\left|h_{k, \tilde{k}}\right|_{\mathbf{k}} & =\left|\left(c_{k, \tilde{k}}+g_{k, \tilde{k}}\right) \overline{\operatorname{sgn} d_{k, \tilde{k}}} \operatorname{sgn} d_{k, \tilde{k}}\right|_{\mathbf{k}} \\
& =\left|\left(c_{k, \tilde{k}} \overline{\operatorname{sgn} d_{k, \tilde{k}}}+g_{k, \tilde{k}} \overline{\operatorname{sgn} d_{k, \tilde{k}}}\right) \operatorname{sgn} d_{k, \tilde{k}}\right|_{\mathbf{k}}
\end{aligned}
$$


We have that

$$
c_{k, \tilde{k}} \overline{\operatorname{sgn} d_{k, \tilde{k}}}=c_{k} \mathbf{e}^{+}\left(\frac{\bar{c}_{k}}{\left|c_{k}\right|} \mathbf{e}^{+}+\frac{\bar{c}_{k}}{\left|c_{k}\right|} \mathbf{e}^{-}\right)=\left|c_{k}\right| \mathbf{e}^{+}+0 \mathbf{e}^{-}=\left|c_{k, \tilde{k}}\right|_{\mathbf{k}}
$$

and, therefore,

$$
\begin{aligned}
\left|h_{k, \tilde{k}}\right|_{\mathbf{k}} & =\left.|| c_{k, \tilde{k}}\right|_{\mathbf{k}}+\left.g_{k, \tilde{k}} \overline{\operatorname{sgn} d_{k, \tilde{k}}}\right|_{\mathbf{k}}\left|\overline{\operatorname{sgn} d_{k, \tilde{k}}}\right|_{\mathbf{k}} \\
& =\left.|| c_{k, \tilde{k}}\right|_{\mathbf{k}}+\left.g_{k, \tilde{k}} \overline{\operatorname{sgn} d_{k, \tilde{k}}}\right|_{\mathbf{k}},
\end{aligned}
$$

so that (3.11) holds with $\overline{\operatorname{sgn} d_{k, \tilde{k}}}$ replacing $\overline{\operatorname{sgn} c_{k, \tilde{k}}}$.

Hence, for $(k, \tilde{k}) \in T$ we fix $P_{k, \tilde{k}}=c_{k, \tilde{k}}$ if both $c_{k}, c_{\tilde{k}}$ are non-zero, and $P_{k, \tilde{k}}=d_{k, \tilde{k}}$ otherwise. In this case the elements of the sequence $P=\left(P_{k, \tilde{k}}\right)_{k, \tilde{k}}$ satisfies (1) and (3) and we get

$$
2 \operatorname{Re}\left(\left|h_{k, \tilde{k}}\right|_{\mathbf{k}}\right) \geq 2 \operatorname{Re}\left(\left|c_{k, \tilde{k}}\right|_{\mathbf{k}}\right)+2 \operatorname{Re}\left(g_{k, \tilde{k}} \overline{P_{k, \tilde{k}}}\right)=\left|c_{k}\right|+\left|c_{\tilde{k}}\right|+2 \operatorname{Re}\left(g_{k, \tilde{k}} \overline{P_{k, \tilde{k}}}\right) .
$$

Thus, we have obtained that for all $(k, \tilde{k}) \in T$ it holds

$$
\left|c_{k}\right|+\left|c_{\tilde{k}}\right|+2 \operatorname{Re}\left(g_{k, \tilde{k}} \overline{P_{k, \tilde{k}}}\right) \leq\left|h_{k}\right|+\left|h_{\tilde{k}}\right|=2 \operatorname{Re}\left(\left|h_{k, \tilde{k}}\right|_{\mathbf{k}}\right) .
$$

For $(k, \tilde{k}) \notin T$ we choose $P_{k, \tilde{k}}=c_{k, \tilde{k}}$ so that (2) is satisfied and by direct calculations (recall, $\left.c_{k, \tilde{k}}=0\right)$ we get

$$
2 \operatorname{Re}\left(h_{k, \tilde{k}}\right)=2 \operatorname{Re}\left(g_{k, \tilde{k}}\right) \geq 2 \operatorname{Re}\left(g_{k, \tilde{k}} \overline{P_{k, \tilde{k}}}\right) .
$$

Hence, we get

$$
\|h\|_{\ell_{1}} \geq\|c\|_{\ell_{1}}+\sum_{(k, \tilde{k}) \in \mathbb{Z}_{\rho}^{2}} 2 \operatorname{Re}\left(g_{k, \tilde{k}} \overline{P_{k, \tilde{k}}}\right),
$$

Using the Plancherel formula for the discrete Fourier transform (see [7]) we have

$$
\sum_{(k, \tilde{k}) \in \mathbb{Z}_{\rho}^{2}} \operatorname{Re}\left(g_{k, \tilde{k}} \overline{P_{k, \tilde{k}}}\right)=\frac{1}{N} \sum_{j=1}^{N} \operatorname{Re}\left(\mathcal{F}_{X} g\left(x_{j}, y_{j}\right) \overline{\mathcal{F}_{X} P\left(x_{j}, y_{j}\right)}\right) .
$$

Taking in account that $g_{k, \tilde{k}} \overline{P_{k, \tilde{k}}}=g_{k} \overline{P_{k}} \mathbf{e}^{+}+g_{\tilde{k}} \overline{P_{\tilde{k}}} \mathbf{e}^{-}$we obtain

$$
\begin{gathered}
\sum_{(k, \tilde{k}) \in \mathbb{Z}_{\rho}^{2}} \operatorname{Re}\left(g_{k, \tilde{k}} \overline{P_{k, \tilde{k}}}\right)=\sum_{(k, \tilde{k}) \in \mathbb{Z}_{\rho}^{2}} \operatorname{Re}\left(g_{k} \overline{P_{k}} \mathbf{e}^{+}+g_{\tilde{k}} \overline{P_{\tilde{k}}} \mathbf{e}^{-}\right) \\
=\sum_{(k, \tilde{k}) \in \mathbb{Z}_{\rho}^{2}} \operatorname{Re}\left(g_{k} \overline{\left.\left[\mathcal{F}_{X}^{*}\right]_{1} \lambda_{1}\right]_{k}} \mathbf{e}^{+}+g_{\tilde{k}} \overline{\left.\left[\mathcal{F}_{X}^{*}\right]_{2} \lambda_{2}\right]_{\tilde{k}}} \mathbf{e}^{-}\right) \\
=\frac{1}{N} \sum_{j=1}^{N} \operatorname{Re}\left(\mathcal{F}_{X} g\left(x_{j}, y_{j}\right) \overline{\lambda\left(x_{j}, y_{j}\right)}\right)=0
\end{gathered}
$$

since $\mathcal{F}_{X} g\left(x_{j}, y_{j}\right)=0, j=1, \ldots, N$. Therefore, $\|h\|_{\ell_{1}} \geq\|c\|_{\ell_{1}}$ and equality holds for $2 \operatorname{Re}\left(g_{k, \tilde{k}} \overline{P_{k, \tilde{k}}}\right)=$ $2 \operatorname{Re}\left(\left|g_{k, \tilde{k}}\right|_{\mathbf{k}}\right)$. Since $\left|P_{k, \tilde{k}}\right|<1$, this forces $g$ to vanish outside $T$. Taking into account the injectivity of $\mathcal{F}_{T X}$ we get that if $\mathcal{F}_{X} g$ vanishes on $X, g$ vanishes identically there and, therefore, $h=c$. This shows that $c$ is the unique minimizer to the problem (3.9). 


\section{Proof of Main Theorems}

We are now in conditions to prove Theorem 3.1. First, we show that for every sequence $c=$ $\left(c_{k, \tilde{k}}\right) \in \ell_{2}\left(\mathbb{Z}_{\rho}^{2}\right)$ with support on $T$ with high probability there exists a sequence $P$ satisfying to Lemma 3.2, (1)-(3).

4.1. Existence with High Probability of a Sequence $P$. We introduce the restriction operator $R_{T}: \ell_{2}\left(\mathbb{Z}_{\rho}^{2}\right) \rightarrow \ell_{2}(T)$, which maps $c \in \ell_{2}\left(\mathbb{Z}_{\rho}^{2}\right)$ into $R_{T} c:=\left(c_{k, \tilde{k}}\right)_{(k, \tilde{k}) \in T}$. Its adjoint $R_{T}^{*}=$ $E_{T}: \ell_{2}(T) \rightarrow \ell_{2}\left(\mathbb{Z}_{\rho}^{2}\right)$ is the operator which extends the restricted sequence $R_{T} c=\left(c_{k, \tilde{k}}\right)_{(k, \tilde{k}) \in T}$ to a sequence $d \in \ell_{2}\left(\mathbb{Z}_{\rho}^{2}\right)$ where $d_{k, \tilde{k}}=c_{k, \tilde{k}}$ for $(k, \tilde{k}) \in T$, and $d_{k, \tilde{k}}=0$ otherwise.

Let us assume that $\mathcal{F}_{T X}^{*} \mathcal{F}_{T X}: \ell_{2}(T) \rightarrow \ell_{2}(T)$ is invertible. This is true almost surely if $N \geq|T|$ since $\mathcal{F}_{T X}$ is injective. Moreover, its inverse is given by the following lemma.

Lemma 4.1 (see [9]). For a non-singular matrix $\mathcal{F}_{T X}^{*} \mathcal{F}_{T X}$ we have

$$
\left(\mathcal{F}_{T X}^{*} \mathcal{F}_{T X}\right)^{-1}=\left[\mathcal{F}_{T X}^{*} \mathcal{F}_{T X}\right]_{1}^{-1} \mathbf{e}^{+}+\left[\mathcal{F}_{T X}^{*} \mathcal{F}_{T X}\right]_{2}^{-1} \mathbf{e}^{-} .
$$

We set $P:=\mathcal{F}_{X}^{*} \mathcal{F}_{T X}\left(\mathcal{F}_{T X}^{*} \mathcal{F}_{T X}\right)^{-1} R_{T}$ sgnc. Clearly, $P$ verifies (1) and (3) of Lemma 3.2, with

$$
\lambda:=\mathcal{F}_{T X}\left(\mathcal{F}_{T X}^{*} \mathcal{F}_{T X}\right)^{-1} R_{T} \operatorname{sgn} c \in \ell_{2}(X) .
$$

It remains to prove that $P$ satisfies (3) with high probability. We begin with the idempotent decomposition of our bicomplex matrices $I=I \mathbf{e}^{+}+I \mathbf{e}^{-}(I$ identity matrix $), E_{T}=E_{T} \mathbf{e}^{+}+E_{T} \mathbf{e}^{-}$, and

$$
\mathcal{F}_{X}^{*} \mathcal{F}_{T X}=\left[\mathcal{F}_{X}^{*} \mathcal{F}_{T X}\right]_{1} \mathbf{e}^{+}+\left[\mathcal{F}_{X}^{*} \mathcal{F}_{T X}\right]_{2} \mathbf{e}^{-}, \quad \mathcal{F}_{T X}^{*} \mathcal{F}_{T X}=\left[\mathcal{F}_{T X}^{*} \mathcal{F}_{T X}\right]_{1} \mathbf{e}^{+}+\left[\mathcal{F}_{T X}^{*} \mathcal{F}_{T X}\right]_{2} \mathbf{e}^{-} .
$$

We introduce the auxiliary operators

$$
\begin{gathered}
H:=N E_{T}-\mathcal{F}_{X}^{*} \mathcal{F}_{T X}: \ell_{2}(T) \rightarrow \ell_{2}\left(\mathbb{Z}_{\rho}^{2}\right), \\
H_{0}:=R_{T} H=N I-\mathcal{F}_{T X}^{*} \mathcal{F}_{T X}: \ell_{2}(T) \rightarrow \ell_{2}(T) .
\end{gathered}
$$

Obviously, $H_{0}$ is self-adjoint, and both operators admit the following idempotent decompositions

$$
\begin{aligned}
& H=\left(N E_{T}-\left[\mathcal{F}_{X}^{*} \mathcal{F}_{T X}\right]_{1}\right) \mathbf{e}^{+}+\left(N E_{T}-\left[\mathcal{F}_{X}^{*} \mathcal{F}_{T X}\right]_{2}\right) \mathbf{e}^{-} \\
& H_{0}=\left(N I-\left[\mathcal{F}_{T X}^{*} \mathcal{F}_{T X}\right]_{1}\right) \mathbf{e}^{+}+\left(N I-\left[\mathcal{F}_{T X}^{*} \mathcal{F}_{T X}\right]_{2}\right) \mathbf{e}^{-} .
\end{aligned}
$$

We remark that we are particularly interested in the entries of these matrices. For the first matrix we have

$$
H=\left[\left(1-\delta_{\ell, k}\right) \sum_{j=1}^{N} e^{\mathbf{i}(\ell-k) x_{j}} \mathbf{e}^{+}+\left(1-\delta_{\tilde{\ell}, \tilde{k}}\right) \sum_{j=1}^{N} e^{\mathbf{i}(\tilde{\ell}-\tilde{k}) y_{j}} \mathbf{e}^{-}\right]_{(\ell, \tilde{\ell}),(k, \tilde{k}) \in \mathbb{Z}_{\rho}^{2}},
$$

which acts on a sequence $c=\left(c_{k, \tilde{k}}\right)$ with support in $T$ as

$$
(H c)_{\ell \tilde{\ell}}=-\sum_{j=1}^{N} \sum_{(k, \tilde{k}) \in T}\left(c_{k} e^{\mathbf{i}(k-\ell) x_{j}} \mathbf{e}^{+}+c_{\tilde{k}} e^{\mathbf{i}(\tilde{k}-\tilde{\ell}) y_{j}} \mathbf{e}^{-}\right)
$$


Using these operators and Lemma 4.1, we get

$$
P=\mathcal{F}_{X}^{*} \mathcal{F}_{T X}\left(\mathcal{F}_{T X}^{*} \mathcal{F}_{T X}\right)^{-1} R_{T} \operatorname{sgn} c=\left(N E_{T}-H\right)\left(N I-H_{0}\right)^{-1} R_{T} \operatorname{sgn} c,
$$

where we recall $H_{0}=R_{T} H$. Since we aim to prove that $P$ satisfy Lemma 3.2, (2), we restrict ourselves to $T^{c}:=\mathbb{Z}_{\rho}^{2} \backslash T$. In this case, $R_{T^{c}} E_{T}=0$ and

$$
P_{k, \tilde{k}}=\left(R_{T^{c}} P\right)_{k, \tilde{k}}=-\left(H\left(N I-H_{0}\right)^{-1} R_{T} \operatorname{sgn} c\right)_{k, \tilde{k}},
$$

for all $(k, \tilde{k}) \in T^{c}$. Since $(1-\gamma)^{-1}=\left(1-\gamma^{n}\right)^{-1}\left(1+\gamma+\cdots+\gamma^{n-1}\right)$ we can write

$$
\left(I-\frac{1}{N} H_{0}\right)^{-1}=\left(I-\left(\frac{1}{N} H_{0}\right)^{n}\right)^{-1} \sum_{m=0}^{n-1}\left(\frac{1}{N} H_{0}\right)^{m}
$$

while by the von Neumann series we have

$$
\left(I-\left(\frac{1}{N} H_{0}\right)^{n}\right)^{-1}=I+\sum_{r=1}^{\infty}\left(\frac{1}{N} H_{0}\right)^{r n}:=I+A_{n}
$$

under the assumption that the last series converges. Therefore, for all $(k, \tilde{k}) \in T^{c}$ we get

$$
P_{k, \tilde{k}}=-\left(\frac{1}{N} H\left(I+A_{n}\right) \sum_{m=0}^{n-1}\left(\frac{1}{N} H_{0}\right)^{m} R_{T} \operatorname{sgn} c\right)_{k, \tilde{k}}=-\left(P^{(1)}+P^{(2)}\right)_{k, \tilde{k}},
$$

where (recall, $\left.H_{0}=R_{T} H\right)$

$$
P^{(1)}=\sum_{m=1}^{n}\left(\frac{1}{N} H R_{T}\right)^{m} \operatorname{sgn} c, \quad P^{(2)}=\frac{1}{N} H A_{n} R_{T}\left(I+\sum_{m=1}^{n-1}\left(\frac{1}{N} H R_{T}\right)^{m}\right) \operatorname{sgn} c .
$$

Our goal is show that the probability of $\left|P_{k, \tilde{k}}\right|<1$ is low for $(k, \tilde{k})$ outside $T$. For that effect, we estimate the probability $\mathbb{P}\left(\sup _{(k, \tilde{k}) \in T^{c}}\left|P_{k, \tilde{k}}\right| \geq 1\right)$.

Let $a_{1}, a_{2}>0$ be non-negative real numbers satisfying to $a_{1}+a_{2}=1$. Then,

$$
\mathbb{P}\left(\sup _{(k, \tilde{k}) \in T^{c}}\left|P_{k, \tilde{k}}\right| \geq 1\right) \leq \mathbb{P}\left(\left\{\sup _{(k, \tilde{k}) \in T^{c}}\left|P_{k, \tilde{k}}^{(1)}\right| \geq a_{1}\right\} \cup\left\{\sup _{(k, \tilde{k}) \in T^{c}}\left|P_{k, \tilde{k}}^{(2)}\right| \geq a_{2}\right\}\right) .
$$

Now, we have

$$
\begin{gathered}
\mathbb{P}\left(\left|P_{k, \tilde{k}}^{(1)}\right| \geq a_{1}\right)=\mathbb{P}\left(\left|\left(\sum_{m=1}^{n}\left(\frac{1}{N} H R_{T}\right)^{m} \operatorname{sgn} c\right)_{k, \tilde{k}}\right| \geq a_{1}\right) \\
\leq \mathbb{P}\left(\sum_{m=1}^{n}\left|\left(\left(\frac{1}{N} H R_{T}\right)^{m} \operatorname{sgn} c\right)_{k, \tilde{k}}\right| \geq a_{1}\right):=\mathbb{P}\left(E_{k, \tilde{k}}\right) .
\end{gathered}
$$


For the term $P^{(2)}$ we obtain

$$
\begin{gathered}
\sup _{(k, \tilde{k}) \in T^{c}}\left|P_{k, \tilde{k}}^{(2)}\right| \leq\left\|P^{(2)}\right\|_{\infty} \\
\leq\left\|\frac{1}{N} H A_{n}\right\|_{\infty}\left(1+\left\|R_{T} \sum_{m=1}^{n-1}\left(\frac{1}{N} H R_{T}\right) \operatorname{sgn} c\right\|_{\ell^{\infty}(T)}\right) .
\end{gathered}
$$

where $\|\cdot\|_{\infty}:=\|\cdot\|_{\ell^{\infty}(T) \rightarrow \ell^{\infty}\left(\mathbb{Z}_{\rho}^{2}\right)}$, and $\ell^{\infty}\left(\mathbb{Z}_{\rho}^{2}\right)$ denote the space of sequences indexed by $\mathbb{Z}_{\rho}^{2}$ with the supremum hyperbolic norm. We have, by (4.10),

$$
\begin{array}{r}
\mathbb{P}\left(\left|\left(\sum_{m=1}^{n-1}\left(\frac{1}{N} H R_{T}\right)^{m} \operatorname{sgn} c\right)_{k, \tilde{k}}\right| \geq a_{1}\right) \leq \mathbb{P}\left(\sum_{m=1}^{n-1}\left|\left(\left(\frac{1}{N} H R_{T}\right)^{m} \operatorname{sgn} c\right)_{k, \tilde{k}}\right| \geq a_{1}\right) \\
\leq \mathbb{P}\left(\sum_{m=1}^{n}\left|\left(\left(\frac{1}{N} H R_{T}\right)^{m} \operatorname{sgn} c\right)_{k, \tilde{k}}\right| \geq a_{1}\right)=\mathbb{P}\left(E_{k, \tilde{k}}\right) .
\end{array}
$$

Now, we analyse the operator norm in (4.11) in terms of the Frobenius norm (see [10] and [8]). First of all, we have

$$
\left\|\frac{1}{N} H A_{n}\right\|_{\infty} \leq\left\|\frac{1}{N} H\right\|_{\infty}\left\|A_{n}\right\|_{\ell^{\infty}(T)}=\left\|\frac{1}{N} H\right\|_{\infty}\left\|\sum_{r=1}^{\infty}\left(\frac{1}{N} H_{0}\right)^{r n}\right\|_{\ell^{\infty}(T)} .
$$

Given a square matrix $B$, with bicomplex entries, it holds for its Frobenius norm defined with respect to the hyperbolic norm, the equality $\|B\|_{F}^{2}:=\operatorname{Re}\left(\operatorname{tr}\left(B B^{*}\right)\right)=\sum_{r, s} 2 \operatorname{Re}\left(\left|B_{r s}\right|_{\mathbf{k}}^{2}\right)$, where $\operatorname{tr}\left(B B^{*}\right)$ denotes the trace of $B B^{*}$. Assume now that

$$
\left\|\left(\frac{1}{N} H_{0}\right)^{n}\right\|_{F} \leq \kappa<1 .
$$

From (4.9) we obtain

$$
\left\|A_{n}\right\|_{F}=\left\|\sum_{r=1}^{\infty}\left(\frac{1}{N} H_{0}\right)^{r n}\right\|_{F} \leq \sum_{r=1}^{\infty}\left\|\left(N^{-1} H_{0}\right)^{n}\right\|_{F}^{r} \leq \sum_{r=1}^{\infty} \kappa^{r}=\frac{\kappa}{1-\kappa} .
$$

Also, by Cauchy-Schwarz inequality we get

$$
\left\|A_{n}\right\|_{\ell^{\infty}(T)}^{2} \leq|T|\left(2 \sup _{r} \sum_{s} \operatorname{Re}\left(\left|\left[A_{n}\right]_{r, s}\right|_{\mathbf{k}}\right)^{2}\right) \leq|T|\left\|A_{n}\right\|_{F}^{2} .
$$

Under the assumptions (4.13), and $\left\|\sum_{m=1}^{n-1}\left(\frac{1}{N} H R_{T}\right)^{m} \operatorname{sgn} c\right\|_{\infty}<a_{1}$, it holds

$$
\sup _{(k, \tilde{k}) \in T^{c}}\left|P_{k \tilde{k}}^{(2)}\right| \leq\left(1+a_{1}\right) \frac{\kappa}{1-\kappa}|T|^{\frac{3}{2}} .
$$

Moreover, if $\frac{\kappa}{1-\kappa} \leq \frac{a_{2}}{1+a_{1}}|T|^{-\frac{3}{2}}$ then, we have $\sup _{(k, \tilde{k}) \in T^{c}}\left|P_{k \tilde{k}}^{(2)}\right| \leq a_{2}$. Also, it implies that $\kappa<1$ and $|T| \geq 1$ (note that if $T=\emptyset$ then $c=0$ and obviously the $\ell_{1}$-minimization problem recovers the sequence). This concludes the proof of the existence with high probability of a sequence $P$ satisfying to the conditions of Lemma 3.2. As an additional consequence, it also ensures that $\mathcal{F}_{T X}$ 
is injective almost surely.

Next, we proceed with the analysis of the above probability bearing in mind that $T$ is a deterministic variable, as seen in Theorem 3.2. We have

$$
\mathbb{P}\left(\sup _{(k, \tilde{k}) \in T^{c}}\left|P_{k, \tilde{k}}\right| \geq 1\right) \leq \sum_{(k, \tilde{k}) \in T^{c}} \mathbb{P}\left(E_{k, \tilde{k}}\right)+\mathbb{P}\left(\left\|\left(\frac{1}{N} H_{0}\right)^{n}\right\|_{F} \geq \kappa\right) .
$$

In conclusion, the probability of the existence of a sequence $P$ depends on the estimates for $\mathbb{P}\left(E_{k \tilde{k}}\right)$ and for $\mathbb{P}\left(\left\|\left(\frac{1}{N} H_{0}\right)^{n}\right\|_{F} \geq \kappa\right)$.

4.2. Analysis of the Powers $H_{0}^{2 n}$. We now compute the expectation of $\left\|H_{0}^{n}\right\|_{F}^{2}$ with respect to the sampling set $\left\{\left(x_{j}, y_{j}\right), j=1, \ldots, N\right\}$.

Lemma 4.2. It holds

$$
\mathbb{E}_{X}\left[\left\|H_{0}^{n}\right\|_{F}^{2}\right]=\sum_{t=1}^{\min \{n, N\}} \frac{N !}{(N-t) !} \sum_{\mathcal{A} \in P(2 n, t)} C(\mathcal{A}, T),
$$

where $P(2 n, t)$ denotes the set of all partitions $A=\left(A_{1}, \ldots, A_{t}\right)$ of the set $1, \ldots, 2 n$ and

$$
C(\mathcal{A}, T):=\sum_{\substack{\left(k_{1}, \tilde{k}_{1}\right), \cdots,\left(k_{2 n}, \tilde{k}_{2 n}\right) \in T \\ k_{r+1} \neq k_{r}, \tilde{k}_{r+1} \neq \tilde{k}_{r} \\ k_{2 n+1}:=k_{1}, \tilde{k}_{2 n+1}:=\tilde{k}_{1}}} \prod_{A \subset \mathcal{A}}\left(\delta\left(\sum_{r \in A}\left(k_{r+1}-k_{r}\right)\right)+\delta\left(\sum_{r \in A}\left(\tilde{k}_{r+1}-\tilde{k}_{r}\right)\right)\right)
$$

Proof. We remember the operator (4.2) with an idempotent decomposition at each entry $[(\ell, \tilde{\ell}),(k, \tilde{k})]$

$$
H_{0}[(\ell, \tilde{\ell}),(k, \tilde{k})]=H_{0,1}(\ell, k) \mathbf{e}^{+}+H_{0,2}(\tilde{\ell}, \tilde{k}) \mathbf{e}^{-} .
$$

Hence, its adjoint is

$$
H_{0}^{*}[(\ell, \tilde{\ell}),(k, \tilde{k})]=\bar{H}_{0,1}(k, \ell) \mathbf{e}^{+}+\bar{H}_{0,2}(\tilde{k}, \tilde{\ell}) \mathbf{e}^{-},
$$

where $\bar{H}_{0,1}, \bar{H}_{0,2}$ represent the matrices with complex conjugated entries. Therefore, at the main diagonal we have

$$
\begin{aligned}
& H_{0}^{2}[(k, \tilde{k}),(k, \tilde{k})]=\sum_{(\ell, \tilde{\ell}) \in T} H_{0}[(k, \tilde{k}),(\ell, \tilde{\ell})] H_{0}^{*}[(\ell, \tilde{\ell}),(k, \tilde{k})] \\
= & \sum_{(\ell, \tilde{\ell}) \in T}\left(H_{0,1}(k, \ell) \mathbf{e}^{+}+H_{0,2}(\tilde{k}, \tilde{\ell}) \mathbf{e}^{-}\right)\left(\bar{H}_{0,1}(k, \ell) \mathbf{e}^{+}+\bar{H}_{0,2}(\tilde{k}, \tilde{\ell}) \mathbf{e}^{-}\right) \\
= & \sum_{j_{1}, j_{2}=1}^{N} \sum_{\substack{(\ell, \tilde{\ell}) \in T \\
\ell \neq k, \tilde{\ell} \neq \tilde{k}}}\left[e^{\mathbf{i}(\ell-k) x_{j_{1}}} e^{\mathbf{i}(k-\ell) x_{j_{2}}} \mathbf{e}^{+}+e^{\mathbf{i}(\tilde{\ell}-\tilde{k}) y_{j_{1}}} e^{\mathbf{i}(\tilde{k}-\tilde{\ell}) y_{j_{2}}} \mathbf{e}^{-}\right] .
\end{aligned}
$$

For the generalization to an arbitrary power $H_{0}^{2 n}=\left(H_{0} H_{0}^{*}\right)^{n}$, we shall calculate its trace as the sum of the entries $\left[\left(k_{1}, \tilde{k}_{1}\right),\left(k_{1}, \tilde{k}_{1}\right)\right]$, and will denote the auxiliary entries $(\ell, \tilde{\ell})$ by $\left(k_{s}, \tilde{k}_{s}\right), s=$ 
$2, \ldots, 2 n$. Hence, (4.18) becomes

$$
\begin{aligned}
& H_{0}^{2}\left[\left(k_{1}, \tilde{k}_{1}\right),\left(k_{1}, \tilde{k}_{1}\right)\right] \\
& =\sum_{j_{1}, j_{2}=1}^{N} \sum_{\left(k_{2}, \tilde{k}_{2}\right) \in T}\left[e^{\mathbf{i}\left(k_{2}-k_{1}\right) x_{j_{1}}} e^{\mathbf{i}\left(k_{1}-k_{2}\right) x_{j_{2}}} \mathbf{e}^{+}+e^{\mathbf{i}\left(\tilde{k}_{2}-\tilde{k}_{1}\right) y_{j_{1}}} e^{\mathbf{i}\left(\tilde{k}_{1}-\tilde{k}_{2}\right) y_{j_{2}}} \mathbf{e}^{-}\right], \\
& k_{2} \neq k_{1}, \tilde{k}_{2} \neq \tilde{k}_{1}
\end{aligned}
$$

while for the $n$-th power of our matrix we get

$$
\begin{aligned}
& H_{0}^{2 n}\left[\left(k_{1}, \tilde{k}_{1}\right),\left(k_{1}, \tilde{k}_{1}\right)\right]=\left(\sum_{(\ell, \tilde{\ell}) \in T} H_{0}\left[\left(k_{1}, \tilde{k}_{1}\right),(\ell, \tilde{\ell})\right] H_{0}^{*}\left[(\ell, \tilde{\ell}),\left(k_{1}, \tilde{k}_{1}\right)\right]\right)^{n} \\
& =\quad \sum H_{0}^{2}\left[\left(k_{1}, \tilde{k}_{1}\right),\left(k_{2}, \tilde{k}_{2}\right)\right] \cdots H_{0}^{2}\left[\left(k_{2 n}, \tilde{k}_{2 n}\right),\left(k_{1}, \tilde{k}_{1}\right)\right] \text {, } \\
& \left(k_{2}, \tilde{k}_{2}\right), \cdots,\left(k_{2 n}, \tilde{k}_{2 n}\right) \in T \\
& k_{2} \neq k_{1}, \tilde{k}_{2} \neq \tilde{k}_{1} \\
& k_{r+1} \neq k_{r}, \tilde{k}_{r+1} \neq \tilde{k}_{r}, \quad r=2, \cdots, 2 n-1 \\
& k_{2 n} \neq k_{1}, \tilde{k}_{2 n} \neq \tilde{k}_{1}
\end{aligned}
$$

and the trace of $H_{0}^{2 n}=\left(H_{0} H_{0}^{*}\right)^{n}$ becomes

$$
\begin{aligned}
& \operatorname{tr} H_{0}^{2 n}=\sum_{\left(k_{1}, \tilde{k}_{1}\right) \in T} H_{0}^{2 n}\left[\left(k_{1}, \tilde{k}_{1}\right),\left(k_{1}, \tilde{k}_{1}\right)\right] \\
& =\quad \sum \quad H_{0}^{2}\left[\left(k_{1}, \tilde{k}_{1}\right),\left(k_{2}, \tilde{k}_{2}\right)\right] \cdots H_{0}^{2}\left[\left(k_{2 n}, \tilde{k}_{2 n}\right),\left(k_{1}, \tilde{k}_{1}\right)\right] \\
& \left(k_{1}, \tilde{k}_{1}\right), \cdots,\left(k_{2 n}, \tilde{k}_{2 n}\right) \in T \\
& k_{r+1} \neq k_{r}, \tilde{k}_{r+1} \neq \tilde{k}_{r}, \quad r=1, \cdots, 2 n \\
& k_{2 n+1}:=k_{1}, \tilde{k}_{2 n+1}:=\tilde{k}_{1} \\
& =\sum_{j_{1}, \cdots, j_{2 n}=1}^{N} \sum_{\left(k_{1}, \tilde{k}_{1}\right), \cdots,\left(k_{2 n}, \tilde{k}_{2 n}\right) \in T}\left[e^{\mathbf{i}\left(k_{r+1}-k_{r}\right) x_{j_{r}}} e^{\mathbf{i}\left(k_{r}-k_{r+1}\right) x_{j_{r+1}}} \mathbf{e}^{+}+e^{\mathbf{i}\left(\tilde{k}_{r+1}-\tilde{k}_{r}\right) y_{j_{r}}} e^{\mathbf{i}\left(\tilde{k}_{r}-\tilde{k}_{r+1}\right) y_{j_{r+1}}} \mathbf{e}^{-}\right] \\
& k_{r+1} \neq k_{r}, \tilde{k}_{r+1} \neq \tilde{k}_{r} \\
& k_{2 n+1}:=k_{1}, \tilde{k}_{2 n+1}:=\tilde{k}_{1} \\
& =\sum_{j_{1}, \cdots, j_{2 n}=1}^{N} \sum_{\left(k_{1}, \tilde{k}_{1}\right), \cdots,\left(k_{2 n}, \tilde{k}_{2 n}\right) \in T} \\
& k_{r+1} \neq k_{r}, \tilde{k}_{r+1} \neq \tilde{k}_{r} \\
& k_{2 n+1}:=k_{1}, \tilde{k}_{2 n+1}:=\tilde{k}_{1} \\
& {\left[e^{\mathbf{i} \sum_{r=1}^{2 n}\left(k_{r+1}-k_{r}\right) x_{j_{r}}} \mathbf{e}^{+}+e^{\mathbf{i}\left(\sum_{r=1}^{2 n} \tilde{k}_{r+1}-\tilde{k}_{r}\right) y_{j_{r}}} \mathbf{e}^{-}\right] .}
\end{aligned}
$$


In consequence, its mean value is given by

$$
\begin{gathered}
\mathbb{E}_{X}\left[\left\|H_{0}^{n}\right\|_{F}^{2}\right]=\mathbb{E}_{X}\left[2 \operatorname{Re}\left(\operatorname{tr}\left(\left(H_{0} H_{0}^{*}\right)^{n}\right)\right)\right] \\
\sum_{\substack{j_{1}=1 \\
\vdots}}^{\substack{\left(k_{1}, \tilde{k}_{1}\right), \cdots,\left(k_{2 n}, \tilde{k}_{2 n}\right) \in T \\
k_{r+1} \neq k_{r}, \tilde{k}_{r+1} \neq \tilde{k}_{r} \\
k_{2 n+1}:=k_{1}, \tilde{k}_{2 n+1}:=\tilde{k}_{1}}} \mathbb{E}_{X}\left[2 \operatorname{Re}\left(e^{\mathbf{i} \sum_{r=1}^{2 n}\left(k_{r+1}-k_{r}\right) x_{j_{r}}} \mathbf{e}^{+}+e^{\mathbf{i}\left(\sum_{r=1}^{2 n} \tilde{k}_{r+1}-\tilde{k}_{r}\right) y_{j_{r}}} \mathbf{e}^{-}\right)\right] . \\
j_{2 n}=1
\end{gathered}
$$

Now, we have to keep in mind that some of the indices $j_{r}$ might coincide, in which case the multiplying terms $\left(k_{r+1}-k_{r}\right)$, etc. should be added. The idea to solve this problem comes from [14] where the author introduced a rearrangement based on set partitions. Here, we follow that idea by associating a partition $\mathcal{A}=\left(A_{1}, \ldots, A_{t}\right)$ of the set $\{1, \ldots, 2 n\}$ to a certain vector $\left(j_{1}, \ldots, j_{2 n}\right)$ such that $j_{r}=j_{r^{\prime}}$ if and only if $r$ and $r^{\prime}$ are contained in the same set $A \subset \mathcal{A}$. This will allow us, in an unambiguous way, to write $j_{A}$ instead of $j_{r}$ if $r \in A$. The independence of the sampling variables leads to

$$
\begin{aligned}
& \mathbb{E}_{X}\left[2 \operatorname{Re}\left(e^{\mathbf{i} \sum_{r=1}^{2 n}\left(k_{r+1}-k_{r}\right) x_{j_{r}}} \mathbf{e}^{+}+e^{\mathbf{i}\left(\sum_{r=1}^{2 n} \tilde{k}_{r+1}-\tilde{k}_{r}\right) y_{j_{r}}} \mathbf{e}^{-}\right)\right] \\
& =\mathbb{E}_{X}\left[2 \operatorname{Re}\left(e^{\mathbf{i} \sum_{A \subset \mathcal{A}} \sum_{r \in A}\left(k_{r+1}-k_{r}\right) x_{j}} \mathbf{e}^{+}+e^{\mathbf{i}\left(\sum_{A \subset \mathcal{A}} \sum_{r \in A}\left(\tilde{k}_{r+1}-\tilde{k}_{r}\right) y_{j} \mathbf{e}^{-}\right)}\right]\right. \\
& =\mathbb{E}_{X}\left[2 \operatorname{Re}\left(\prod_{A \subset \mathcal{A}} e^{\mathbf{i} \sum_{r \in A}\left(k_{r+1}-k_{r}\right) x_{j}} \mathbf{e}^{+}+\prod_{A \subset \mathcal{A}} e^{\mathbf{i}\left(\sum_{r \in A}\left(\tilde{k}_{r+1}-\tilde{k}_{r}\right) y_{j_{A}} \mathbf{e}^{-}\right.}\right)\right] \\
& =\prod_{A \subset \mathcal{A}} \mathbb{E}_{X}\left[2 \operatorname{Re}\left(e^{\mathbf{i} \sum_{r \in A}\left(k_{r+1}-k_{r}\right) x_{j}} \mathbf{e}^{+}+e^{\mathbf{i}\left(\sum_{r \in A}\left(\tilde{k}_{r+1}-\tilde{k}_{r}\right) y_{j} A\right.} \mathbf{e}^{-}\right)\right]
\end{aligned}
$$

Finally, taking into account that the variables $\left(x_{j_{A}}, x_{j_{A}}\right)$ have uniform distribution on $[0,2 \pi]^{2}$ we conclude that the expectation value is

$$
\begin{gathered}
\mathbb{E}_{X}\left[2 \operatorname { R e } \left(e^{\mathbf{i} \sum_{r \in A}\left(k_{r+1}-k_{r}\right) x_{j}} \mathbf{e}^{+}+e^{\left.\mathbf{i}\left(\sum_{r \in A}\left(\tilde{k}_{r+1}-\tilde{k}_{r}\right) y_{j_{A}} \mathbf{e}^{-}\right)\right]}\right.\right. \\
=\mathbb{E}_{X}\left[e^{\mathbf{i} \sum_{r \in A}\left(k_{r+1}-k_{r}\right) x_{j_{A}}}\right]+\mathbb{E}_{X}\left[e^{\mathbf{i}\left(\sum_{r \in A}\left(\tilde{k}_{r+1}-\tilde{k}_{r}\right) y_{j_{A}}\right.}\right] \\
=\frac{1}{2 \pi} \int_{0}^{2 \pi} e^{\mathbf{i} \sum_{r \in A}\left(k_{r+1}-k_{r}\right) x} d x+\frac{1}{2 \pi} \int_{0}^{2 \pi} e^{\mathbf{i}\left(\sum_{r \in A}\left(\tilde{k}_{r+1}-\tilde{k}_{r}\right) y\right.} d y \\
=\delta\left(\sum_{r \in A}\left(k_{r+1}-k_{r}\right)\right)+\delta\left(\sum_{r \in A}\left(\tilde{k}_{r+1}-\tilde{k}_{r}\right)\right)
\end{gathered}
$$

If $A \subset \mathcal{A}$ contains only one element then (4.20) vanishes taking in account our conditions $k_{r+1} \neq$ $k_{r}, \tilde{k}_{r+1} \neq \tilde{k}_{r}$. Hence, $|A|>1$ for all $A \in \mathcal{A}$, i.e., partitions in $P(2 n, t)$. Furthermore, note that for each $t$ the number of vectors $\left(j_{A_{1}}, \ldots, j_{A_{t}}\right) \in\{1, \ldots, N\}^{t}$ with different entries is precisely $N(N-1) \cdots(N-t+1)=\frac{N !}{(N-t) !}$ if $N \geq t$ and 0 if $N<t$.

4.3. Further Necessary Estimates. In order to obtain estimates for $\mathbb{P}\left(E_{k, \tilde{k}}\right)$ in $(4.16)$ we require a priori estimations for the expectation of $\operatorname{Re}\left(\left|\left(\left(H R_{T}\right)^{m} \operatorname{sgn}(c)\right)_{k, \tilde{k}}\right|_{\mathbf{k}}^{2 K}\right)$. 
Lemma 4.3. Let $c:=c_{1} \mathbf{e}^{+}+c_{2} \mathbf{e}^{-} \in \ell_{2}\left(\mathbb{Z}_{\rho}^{2}\right)$ be a sequence with support $\operatorname{supp} c=T$. Then, for every $(k, \tilde{k}) \in \mathbb{Z}_{\rho}^{2}$ it holds

$$
\begin{aligned}
& \mathbb{E}_{X}\left[2 \operatorname{Re}\left(\left|\left(\left(H R_{T}\right)^{m} \operatorname{sgn}(c)\right)_{k, \tilde{k}}\right|_{\mathbf{k}}^{2 K}\right)\right] \\
& \leq \sum_{t=1}^{\min \{m K, N\}} \frac{N !}{(N-t) !} \sum_{\mathcal{A} \in P(2 m K, t)} \sum_{\left(k_{1}^{(1)}, \tilde{k}_{1}^{(1)}\right), \ldots,\left(k_{m}^{(1)}, \tilde{k}_{m}^{(1)}\right) \in T} \times \\
& \left(k_{1}^{(2 K)}, \tilde{k}_{1}^{(2 K)}\right), \ldots,\left(k_{m}^{(2 K)}, \tilde{k}_{m}^{(2 K)}\right) \in T \\
& k_{j-1}^{(p)} \neq k_{j}^{(p)}, \tilde{k}_{j-1}^{(p)} \neq \tilde{k}_{j}^{(p)}, j \in[m] \\
& \prod_{A \subset \mathcal{A}}\left[\delta\left(\sum_{(r, p) \in A}(-1)^{p}\left(k_{r}^{(p)}-k_{r-1}^{(p)}\right)\right)+\delta\left(\sum_{(r, p) \in A}(-1)^{p}\left(\tilde{k}_{r}^{(p)}-\tilde{k}_{r-1}^{(p)}\right)\right)\right]
\end{aligned}
$$

with $\left(k_{0}^{(p)}, \tilde{k}_{0}^{(p)}\right):=(k, \tilde{k}), p=1, \ldots, 2 K$. Hereby, we identified the partitions of $[2 m K]$ in $P(2 m K, t)$ with partitions of $[2 K] \times[m]$ in $\mathcal{A}$.

Proof. Again, we recall the idempotent decomposition

$$
\begin{gathered}
\left|\left(\left(H R_{T}\right)^{m} \operatorname{sgn} c\right)_{k \tilde{k}}\right|_{\mathbf{k}}^{2 K}=\left|\left(\left(H_{1} R_{T}\right)^{m} \operatorname{sgn} c_{1}\right)_{k} \mathbf{e}^{+}+\left(\left(H_{2} R_{T}\right)^{m} \operatorname{sgn} c_{2}\right)_{\tilde{k}} \mathbf{e}^{-}\right|_{\mathbf{k}}^{2 K} \\
=\left(\left|\left(\left(H_{1} R_{T}\right)^{m} \operatorname{sgn} c_{1}\right)_{k}\right| \mathbf{e}^{+}+\left|\left(\left(H_{2} R_{T}\right)^{m} \operatorname{sgn} c_{2}\right)_{\tilde{k}}\right| \mathbf{e}^{-}\right)^{2 K} \\
=\left|\left(\left(H_{1} R_{T}\right)^{m} \operatorname{sgn} c_{1}\right)_{k}\right|^{2 K} \mathbf{e}^{+}+\left|\left(\left(H_{2} R_{T}\right)^{m} \operatorname{sgn} c_{2}\right)_{\tilde{k}}\right|^{2 K} \mathbf{e}^{-} .
\end{gathered}
$$

As the treatment of both terms in the idempotent decomposition (4.21) is identical we will omit the second case.

Take $\sigma_{1}:=\operatorname{sgn} c_{1}$. For the first term we have

$$
\left(\left(H_{1} R_{T}\right)^{m} \sigma_{1}\right)_{k}=(-1)^{m} \sum_{j_{1}, \ldots, j_{m}=1}^{N} \sum_{\substack{k_{1}, k_{2}, \ldots, k_{m} \in T_{1} \\ k_{r-1} \neq k_{r}, r=1, \ldots, m}} \sigma_{1}\left(k_{m}\right)\left(e^{\mathbf{i}\left(k_{m}-k_{m-1}\right) x_{j_{m}}} \cdots e^{\mathbf{i}\left(k_{1}-k_{0}\right) x_{j_{1}}}\right),
$$

with $k_{0}:=k$ and $T_{1}=\operatorname{Proj}_{1} T$, the set of all first coordinates of $T$. Thus,

$$
\begin{gathered}
=\sum_{\substack{j_{1}^{(1)}, \ldots, j_{m}^{(1)}=1 \\
j_{1}^{(2)}, \ldots, j_{m}^{(2)}=1}} \sum_{\substack{k_{1}^{(1)}, \ldots, k_{m}^{(1)} \in T_{1} \\
k_{1}^{(2)}, \ldots, k_{m}^{(2)} \in T_{1} \\
k_{j-1}^{(p)} \neq k_{j}^{(p)}, j \in[m], p=1,2}} \sigma_{1}\left(k_{m}^{(1)}\right) \overline{\sigma_{1}\left(k_{m}^{(2)}\right)} \\
\times e^{\mathbf{i} \sum_{r=1}^{m}\left(k_{r}^{1(1)}-k_{r-1}^{1(1)}\right) x_{j_{r}(1)}} e^{-\mathbf{i} \sum_{r=1}^{m}\left(k_{r}^{1(2)}-k_{r-1}^{1(2)}\right) x_{j_{r}}^{(2)}}
\end{gathered}
$$


where $k_{0}^{(1)}=k_{0}^{(2)}=k_{0}=k$. For the $2 K$-th power we obtain

$$
\begin{aligned}
& \left|\left(\left(H_{1} R_{T}\right)^{m} \sigma_{1}\right)_{k}\right|^{2 K}=\sum_{j_{1}^{(1)}, \ldots, j_{m}^{(1)}=1}^{N} \sum_{k_{1}^{(1)}, \ldots, k_{m}^{(1)} \in T_{1}} \sigma_{1}\left(k_{m}^{(1)}\right) \overline{\sigma_{1}\left(k_{m}^{(2)}\right) \times} \\
& j_{1}^{(2 K)}, \ldots, j_{m}^{(2 K)}=1 \quad k_{1}^{(2 K)}, \ldots, k_{m}^{(2 K)} \in T_{1} \\
& k_{j-1}^{(p)} \neq k_{j}^{(p)}, j \in[m], p=1, \ldots, 2 K \\
& \cdots \sigma_{1}\left(k_{m}^{(2 K-1)}\right) \overline{\sigma_{1}\left(k_{m}^{(2 K)}\right)} e^{\mathrm{i}\left(\sum_{p=1}^{2 K}(-1)^{p} \sum_{r=1}^{m}\left(k_{r}^{(p)}-k_{r-1}^{(p)}\right) x_{j_{r}(p)}\right)}
\end{aligned}
$$

with $k_{0}^{(p)}=k$, for $p=1, \ldots, 2 K$.

From (4.21) we obtain

$$
\begin{aligned}
& \mathbb{E}_{X}\left[2 \operatorname{Re}\left(\left|\left(\left(H R_{T}\right)^{m} \operatorname{sgn}(c)\right)_{k, \tilde{k}}\right|_{\mathbf{k}}^{2 K}\right)\right]=\mathbb{E}_{X}\left[\left|\left(\left(H_{1} R_{T}\right)^{m} \operatorname{sgn} c_{1}\right)_{k}\right|^{2 K}+\left|\left(\left(H_{2} R_{T}\right)^{m} \operatorname{sgn} c_{2}\right)_{\tilde{k}}\right|^{2 K}\right]
\end{aligned}
$$

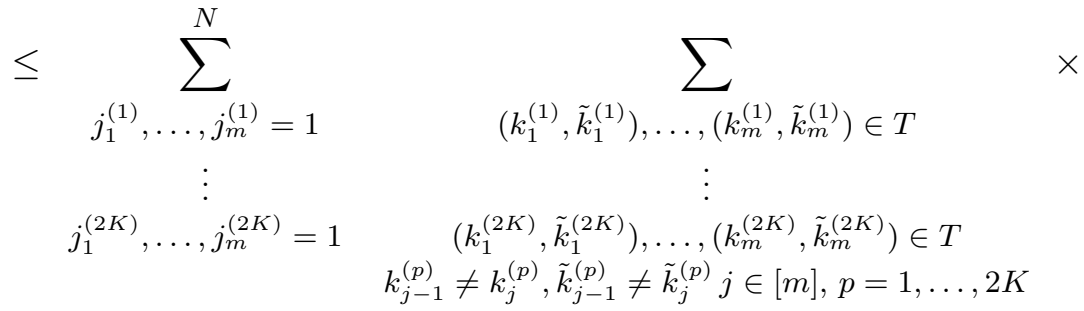

$$
\begin{aligned}
& \left(\mathbb{E}_{X}\left[e^{\mathbf{i}\left(\sum_{p=1}^{2 K}(-1)^{p} \sum_{r=1}^{m}\left(k_{r}^{(p)}-k_{r-1}^{(p)} x_{j_{r}}(p)\right)\right.}\right]+\mathbb{E}_{X}\left[e^{\mathbf{i}\left(\sum_{p=1}^{2 K}(-1)^{p} \sum_{r=1}^{m}\left(\tilde{k}_{r}^{(p)}-\tilde{k}_{r-1}^{(p)}\right) y_{j_{r}}^{(p)}\right)}\right]\right),
\end{aligned}
$$

since $\left|\sigma_{k, \tilde{k}}\right|=1$ for all $(k, \tilde{k}) \in T$.

We further analyze the obtained expected value. As in the proof of Lemma 4.2 we have to take into account that some of the indices $j_{r}^{(p)}$ might coincide. This affords to introduce some additional notation. Let $\left(j_{r}^{(p)}\right)_{r=1, \ldots, m}^{p=1, \ldots, 2 K} \subset\{1, \ldots, N\}^{2 m K}$ be some vector of indices and let $\mathcal{A}=$ $\left(A_{1}, \ldots, A_{t}\right), A_{i} \subset\{1, \ldots, m\} \times\{1, \ldots, 2 K\}$ be a corresponding partition such that $(r, p)$ and $\left(r^{\prime}, p^{\prime}\right)$ are contained in the same block if and only if $j_{r}^{(p)}=j_{r^{\prime}}^{\left(p^{\prime}\right)}$. For some $A \in \mathcal{A}$ we may unambiguously write $j_{A}$ instead of $j_{r}^{(p)}$ if $(r, p) \in A$. Like in (4.19), using that all are different and that the variables $\left(x_{j_{A}}, y_{j_{a}}\right)$ are independent we may write the expectation in (4.22) as

$$
\begin{aligned}
& \mathbb{E}_{X}\left[e^{\mathbf{i}\left(\sum_{p=1}^{2 K}(-1)^{p} \sum_{r=1}^{m}\left(k_{r}^{(p)}-k_{r-1}^{(p)} x_{j_{r}}(p)\right)\right.}\right]+\mathbb{E}_{X}\left[e^{\mathrm{i}\left(\sum_{p=1}^{2 K}(-1)^{p} \sum_{r=1}^{m}\left(\tilde{k}_{r}^{(p)}-\tilde{k}_{r-1}^{(p)}\right) y_{j_{r}}(p)\right)}\right] \\
= & \prod_{A \subset \mathcal{A}}\left(\mathbb{E}_{X}\left[e^{\mathrm{i}\left(\sum_{(r, p) \in A}(-1)^{p}\left(k_{r}^{(p)}-k_{r-1}^{(p)}\right) x_{j_{A}}\right)}\right]+\mathbb{E}_{X}\left[e^{\left.\mathrm{i}\left(\sum_{(r, p) \in A}(-1)^{p}\left(\tilde{k}_{r}^{(p)}-\tilde{k}_{r-1}^{(p)}\right) y_{j_{A}}\right)\right]}\right)\right. \\
= & \prod_{A \subset \mathcal{A}}\left(\delta\left(\sum_{(r, p) \in A}(-1)^{p}\left(k_{r}^{(p)}-k_{r-1}^{(p)}\right)\right)+\delta\left(\sum_{(r, p) \in A}(-1)^{p}\left(\tilde{k}_{r}^{(p)}-\tilde{k}_{r-1}^{(p)}\right)\right)\right) .
\end{aligned}
$$


Once again, if $A \subset \mathcal{A}$ contains only one element then the last expression vanishes due to $k_{r}^{(p)} \neq k_{r-1}^{(p)}$ or $\tilde{k}_{r}^{(p)} \neq \tilde{k}_{r-1}^{(p)}$. Thus, we only need to consider partitions $\mathcal{A} \in P(2 m K, t)$. Now, we can rewrite the inequality in (4.22) as

$$
\begin{aligned}
& \mathbb{E}_{X}\left[2 \operatorname{Re}\left(\left|\left(\left(H R_{T}\right)^{m} \operatorname{sgn}(c)\right)_{k, \tilde{k}}\right|_{\mathbf{k}}^{2 K}\right)\right] \\
& \leq \sum_{t=1}^{m K} \sum_{\mathcal{A} \in P(2 m K, t)} \sum_{\substack{j_{(1)}, \ldots, j_{(t)}=1 \\
\text { all different }}}^{N} \sum_{\left(k_{1}^{(1)}, \tilde{k}_{1}^{(1)}\right), \ldots,\left(k_{m}^{(1)}, \tilde{k}_{m}^{(1)}\right) \in T} \\
& \left(k_{1}^{(2 K)}, \tilde{k}_{1}^{(2 K)}\right), \ldots,\left(k_{m}^{(2 K)}, \tilde{k}_{m}^{(2 K)}\right) \in T \\
& k_{j-1}^{(p)} \neq k_{j}^{(p)}, \tilde{k}_{j-1}^{(p)} \neq \tilde{k}_{j}^{(p)} j \in[m], p=1, \ldots, 2 K \\
& \prod_{A \subset \mathcal{A}}\left(\delta\left(\sum_{(r, p) \in A}(-1)^{p}\left(k_{r}^{(p)}-k_{r-1}^{(p)}\right)\right)+\delta\left(\sum_{(r, p) \in A}(-1)^{p}\left(\tilde{k}_{r}^{(p)}-\tilde{k}_{r-1}^{(p)}\right)\right)\right) \\
& =\sum_{t=1}^{\min \{m K, N\}} \frac{N !}{(N-t) !} \sum_{\mathcal{A} \in P(2 m K, t)} B(\mathcal{A}, T),
\end{aligned}
$$

where

$$
\begin{aligned}
& B(\mathcal{A}, T):=\quad \sum \times \\
& \left(k_{1}^{(1)}, \tilde{k}_{1}^{(1)}\right), \ldots,\left(k_{m}^{(1)}, \tilde{k}_{m}^{(1)}\right) \in T \\
& \left(k_{1}^{(2 K)}, \tilde{k}_{1}^{(2 K)}\right), \ldots,\left(k_{m}^{(2 K)}, \tilde{k}_{m}^{(2 K)}\right) \in T \\
& k_{j-1}^{(p)} \neq k_{j}^{(p)}, \tilde{k}_{j-1}^{(p)} \neq \tilde{k}_{j}^{(p)} j \in[m], p=1, \ldots, 2 K \\
& \prod_{A \subset \mathcal{A}}\left(\delta\left(\sum_{(r, p) \in A}(-1)^{p}\left(k_{r}^{(p)}-k_{r-1}^{(p)}\right)\right)+\delta\left(\sum_{(r, p) \in A}(-1)^{p}\left(\tilde{k}_{r}^{(p)}-\tilde{k}_{r-1}^{(p)}\right)\right)\right) .
\end{aligned}
$$

4.4. Proof of Theorem 3.1. The goal is to complete the proof of Theorem 3.1 with the help of Lemma 4.2 and subsequent results.

Proof. First, we take a closer look into expression (4.2) for $C(\mathcal{A}, T)$, where $\mathcal{A} \in P(2 n, t)$. Here, the indices $\left(\left(k_{1}, \tilde{k}_{1}\right), \ldots,\left(k_{2 n}, \tilde{k}_{2 n}\right)\right) \in T_{1}^{2 n} \times T_{2}^{2 n}$ are subjected to the $|\mathcal{A}|=t$ linear constraints $\sum_{r \in A}\left(k_{r+1}-k_{r}\right)=0$ and $\sum_{r \in A}\left(\tilde{k}_{r+1}-\tilde{k}_{r}\right)=0$ for all $A \in \mathcal{A}$. These constraints are independent except for $\sum_{r=1}^{2 n}\left(k_{r+1}-k_{r}\right)=0$ and $\sum_{r=1}^{2 n}\left(\tilde{k}_{r+1}-\tilde{k}_{r}\right)=0$. Thus, we get for (4.2) the estimate

$$
C(\mathcal{A}, T) \leq|T|^{2 n-t+1} \leq M^{2 n-t+1},
$$


in terms of the sparsity $M$. We remark that in Theorem $3.1 T$ is not random, so that $\mathbb{E}=\mathbb{E}_{X}$. By Lemma 4.2 we obtain

$$
\mathbb{E}\left[\left\|H_{0}^{n}\right\|_{F}^{2}\right] \leq \sum_{t=1}^{\min \{n, N\}} \frac{N !}{(N-t) !} \sum_{\mathcal{A} \in P(2 n, t)}|T|^{2 n-t+1} \leq M^{2 n+1} \sum_{t=1}^{n}\left(\frac{N}{M}\right)^{t} S_{2}(2 n, t),
$$

where $S_{2}(n, t)=|P(2 n, t)|$ are the associated Stirling numbers of the second kind. We define the auxiliar function $F_{n}, n \in \mathbb{N}$, by $F_{n}(\theta):=\sum_{k=1}^{[n / 2]} S_{2}(n, k) \theta^{k}$, and we set $\theta=\frac{N}{M}$. Then,

$$
\begin{gathered}
\mathbb{P}\left(\left\|\left(\frac{1}{N} H_{0}\right)^{n}\right\|_{F} \geq \kappa\right)=\mathbb{P}\left(\left\|\left(\frac{1}{N} H_{0}\right)^{n}\right\|_{F}^{2} \geq \kappa^{2}\right) \leq \kappa^{-2} \mathbb{E}\left[\left\|\left(\frac{1}{N} H_{0}\right)^{n}\right\|_{F}^{2}\right] \\
\leq \kappa^{-2} M \theta^{-2 n} F_{2 n}(\theta)=\kappa^{-2} M G_{2 n}(\theta) .
\end{gathered}
$$

So, $\left\|\left(\frac{1}{N} H_{0}\right)^{n}\right\|_{F} \leq \kappa<1$ implies that $\left(I-\left(\frac{1}{N} H_{0}\right)^{n}\right)$ is invertible by the von Neumann series and, therefore, also $\left[\mathcal{F}_{T X}^{*} \mathcal{F}_{T X}\right]=N\left(I-\frac{1}{N} H_{0}\right)$ is invertible. In particular, we conclude that $\mathcal{F}_{T X}$ is injective. This proves that the injectivity condition in Lemma (3.2) is satisfied automatically with a probability that can be derived from the estimation above.

Now, we turn our attention to the terms $\mathbb{P}\left(E_{k \tilde{k}}\right)$. By Lemma 4.3 we need to bound $B(\mathcal{A}, T)$, i.e., the number of vectors $\left(k_{j}^{(p)}, \tilde{k}_{j}^{(p)}\right) \in T^{2 m K}$ satisfying $\sum_{(r, p) \in A}(-1)^{p}\left(k_{r}^{(p)}-k_{r-1}^{(p)}\right)=0$ and $\sum_{(r, p) \in A}(-1)^{p}\left(\tilde{k}_{r}^{(p)}-\tilde{k}_{r-1}^{(p)}\right)=0$ for all $A \in \mathcal{A}$ with $\mathcal{A} \in P(2 m K, t)$. As these are $t$ independent linear constraints the number of these indices is bounded from above by $|T|^{2 m K-t} \leq M^{2 m K-t}$. Thus, taking $\theta=\frac{N}{M}$, we obtain (similar as above)

$$
\begin{aligned}
\mathbb{E}\left[\left|\left(\left(\frac{1}{N} H R_{T}\right)^{m} \operatorname{sgn} c\right)_{k \tilde{k}}\right|^{2 K}\right] & \leq \sum_{t=1}^{m K} N^{t} S_{2}(2 m K, t) M^{2 m K-t} \\
& =M^{2 m K} F_{2 m K}(\theta) .
\end{aligned}
$$

Now, let $K_{1}, \cdots, K_{n}$ be natural numbers and choose $\beta>0$ such that $\sum_{m=1}^{n} \beta^{n / K_{m}}=a_{1}$ (where $a_{1}$ is as in (4.10)). Then, for every $(k, \tilde{k}) \in T$ it holds for $\mathbb{P}\left(E_{k, \tilde{k}}\right)$ that

$$
\begin{aligned}
\mathbb{P}\left(E_{k, \tilde{k}}\right) & =\mathbb{P}\left(\sum_{m=1}^{n}\left|\left(\left(N^{-1} H R_{T}\right)^{m} \operatorname{sgn} c\right)_{k, \tilde{k}}\right| \geq a_{1}\right) \\
& \leq \sum_{m=1}^{n} \mathbb{P}\left(N^{-m}\left|\left(\left(H R_{T}\right)^{m} \operatorname{sgn} c\right)_{k, \tilde{k}}\right| \geq \beta^{n / K_{m}}\right) \\
& =\sum_{m=1}^{n} \mathbb{P}\left(N^{-2 m K_{m}}\left|\left(\left(H R_{T}\right)^{m} \operatorname{sgn} c\right)_{k, \tilde{k}}\right|^{2 K_{m}} \geq \beta^{2 n}\right) .
\end{aligned}
$$

Since $\mathbb{P}(|x| \geq \gamma)=\mathbb{P}\left(\frac{1}{\gamma}|x| \geq 1\right) \leq \mathbb{E}\left[\frac{1}{\gamma}|x|\right]=\frac{1}{\gamma} \mathbb{E}[|x|],(\gamma>0)$, we conclude

$$
\begin{aligned}
\mathbb{P}\left(E_{k, \tilde{k}}\right) & \leq \sum_{m=1}^{n} \mathbb{P}\left(N^{-2 m K_{m}}\left|\left(\left(H R_{T}\right)^{m} \operatorname{sgn} c\right)_{k, \tilde{k}}\right|^{2 K_{m}} \geq \beta^{2 n}\right) \\
& \leq \beta^{-2 n} \sum_{m=1}^{n} N^{-2 m K_{m}} \mathbb{E}\left[\left|\left(\left(H R_{T}\right)^{m} \operatorname{sgn} c\right)_{k, \tilde{k}}\right|^{2 K_{m}}\right] .
\end{aligned}
$$


From (4.24) we finally obtain

$$
\mathbb{P}\left(E_{k, \tilde{k}}\right) \leq \beta^{-2 n} \sum_{m=1}^{n}\left(\frac{M}{N}\right)^{2 m K_{m}} F_{2 m K_{m}}\left(\frac{N}{M}\right)=\beta^{-2 n} \sum_{m=1}^{n} \theta^{-2 m K_{m}} F_{2 m K_{m}}(\theta) .
$$

Let $\mathbb{P}$ (failure) denote the probability that an exact reconstruction of $f$ by $\ell_{1}$-minimization fails. By Lemma 4.2, it follows

$$
\begin{gathered}
\mathbb{P}(\text { failure }) \leq \mathbb{P}\left(\left\{\mathcal{F}_{T X} \text { is not injective }\right\} \cup\left\{\sup _{(k, \tilde{k}) \in T^{c}}\left|P_{k \tilde{k}}\right| \geq 1\right\}\right) \\
\leq \sum_{(k, \tilde{k}) \in \mathbb{Z}_{\rho}^{2}} \mathbb{P}\left(E_{k \tilde{k}}\right)+\mathbb{P}\left(\left\|\left(N^{-1} H_{0}\right)^{n}\right\| \geq \kappa\right) \leq D \beta^{-2 n} \sum_{m=1}^{n} G_{2 m K_{m}}(\theta)+\kappa^{-2} M G_{2 n}(\theta)
\end{gathered}
$$

under the conditions $a:=a_{1} \sum_{m=1}^{n} \beta^{n / K_{m}}<1, a_{2}=1-a$, and

$$
\frac{\kappa}{1-\kappa} \leq \frac{a_{2}}{1+a_{1}} M^{-3 / 2}=\frac{1-a}{1+a} M^{-3 / 2}
$$

Let us remarks that, given $n$, it is reasonable to take $K_{m} \approx m / n, m=1, \ldots, n$. Then, $\beta$ is chosen quite close to the maximal value such that $a_{1}=\sum_{m=1}^{n} \beta^{n / K_{m}}<1$. By our choice of $K_{m}$ we approximately get

$$
\sum_{m=1}^{n} \beta^{n / K_{m}} \approx \sum_{m=1}^{n} \beta^{m} \approx \frac{\beta}{1-\beta} .
$$

Thus, the optimal $\beta$ will always be close to $1 / 2$.

4.5. Proof of Theorem 3.2. The proof of the Theorem 3.2 is the same as in [14], due to the fact that it depends only on the set partition and it does not enter into account with the algebraic structure of the bicomplex numbers. Therefore, the proof of this theorem is essentially the same as in as [14]. For the sake of convenience for the reader we present it in this subsection.

Proof. We will refine the probability bound (3.7) of Theorem 3.1 in order to obtain Theorem 3.2. First, we proof that the associated Stirling numbers satisfy the estimate

$$
S_{2}(n, k) \leq(3 n / 2)^{n-k} \text { for all } k=1, \ldots,\lfloor n / 2\rfloor .
$$

Indeed, $S_{2}(1, k)=0$ and $S_{2}(2,1)=1$. By induction over $n$ and using the recursion formula $((2.3)$ in Set Partitions), namely

$$
S_{2}(n, k)=k S_{2}(n-1, k)+(n-1) S_{2}(n-1, k-1),
$$

it follows

$$
\begin{aligned}
S_{2}(n, k) & =k S_{2}(n-1, k)+(n-1) S_{2}(n-2, k-1) \\
& \leq k(3(n-1) / 2)^{n-k-1}+(n-1)(3 n / 2-3)^{n-k-1} \leq(n-1+k)(3 n / 2)^{n-k-1} \\
& \leq(3 n / 2)^{n-k},
\end{aligned}
$$


since $(n-1+k) \leq 3 n / 2$. Plugging (4.27) into the definition of $G_{2 n}$ yields

$$
\begin{aligned}
G_{2 n}(\theta) & =\theta^{-2 n} \sum_{k=1}^{n} S_{2}(2 n, k) \theta^{k} \leq \theta^{-2 n} \sum_{k=1}^{n}(3 n)^{2 n-k} \theta^{k}=(3 n / \theta)^{2 n} \sum_{k=1}^{n}(\theta / 3 n)^{k} \\
& =(3 n / \theta)^{2 n-1} \frac{1-(\theta / 3 n)^{n}}{1-(\theta / 3 n)}=(3 n / \theta)^{n} \frac{1-(3 n / \theta)^{n}}{1-3 n / \theta} \\
& \leq \frac{\delta^{n}}{1-\delta}
\end{aligned}
$$

for $n$ chosen in such way that $3 n / \theta \leq \delta<1$. Now consider the term $D \beta^{-2 n} \sum_{m=1}^{n} G_{2 m K_{m}}$ from the probability bound (3.7). We choose $K_{m}=r(n / m)$ where $r(x)$ denotes the function that rounds $x$ to the nearest integer. Then,

$$
m K_{m} \in\{\lceil 2 n / 3\rceil, \ldots,\lfloor 4 n / 3\rfloor\}, \quad m \in\{1, \ldots, n\},
$$

and, thus,

$$
\sum_{k=1}^{n} G_{2 m K_{m}}(\theta) \leq \max _{k \in\{\lceil 2 n / 3\rceil, \ldots,\lfloor 4 n / 3\rfloor\}} G_{2 k}(\theta) \leq \max _{k \in\{\lceil 2 n / 3\rceil, \ldots,\lfloor 4 n / 3\rfloor\}} \frac{\delta^{k}}{1-\delta} \leq n \frac{\delta^{2 n / 3}}{1-\delta}
$$

provided $3 k / \theta \leq \delta$ for all $k \in\{\lceil 2 n / 3\rceil, \ldots,\lfloor 4 n / 3\rfloor\}$. This yields

$$
D \beta^{-2 n} \sum_{m=1}^{n} G_{2 m K_{m}}(\theta) \leq D n \frac{1}{1-\delta}\left(\beta^{-3} \delta\right)^{2 n / 3} .
$$

In order to make this expression small enough we choose $\delta:=\beta^{3} e^{-3 \tau / 2}$, for some $\tau>0$. Then,

$$
D \beta^{-2 n} \sum_{m=1}^{n} G_{2 m K_{m}}(\theta) \leq \frac{D n}{1-\delta} e^{-n \tau} .
$$

The last term in this inequality is smaller than $\epsilon / 2$ if

$$
\ln (D / \epsilon) \leq n \tau+\ln \left(\frac{1-\delta}{2 n}\right)
$$

Let us assume $n \geq S$. Then,

$$
n \tau+\ln \left(\frac{1-\delta}{2 n}\right) \geq n\left(\tau+\frac{\ln \left(\frac{1-\delta}{2 S}\right)}{S}\right) .
$$

Since $3 n / \theta \leq \delta$, we choose now

$$
n=\lfloor\delta \theta / 4\rfloor \geq \frac{S-1}{S} \delta \theta / 4=\frac{S-1}{4 S} \beta^{3} e^{-3 \tau / 2} \theta,
$$

and for those chosen values, we obtain a constant $Q(\beta, \tau, S)$ such that

$$
n \tau+\ln \left(\frac{1-\delta}{2 n}\right) \geq Q(\beta, \tau, S) \theta
$$

Moreover,

$$
D \beta^{-2 n} \sum_{m=1}^{n} G_{2 m K_{m}}(\theta) \leq \epsilon / 2 \quad \text { if } \quad \theta \geq \frac{\ln (D / \epsilon)}{Q(\beta, \tau, S)}
$$


Recalling that $\theta=M / N$ it follows that there exists a constant $C_{1}$ such that $D \beta^{-2 n} \sum_{m=1}^{n} G_{2 m K_{m}}(\theta) \leq$ $\epsilon / 2$ provided

$$
N \geq C_{1} M \ln (D / \epsilon)
$$

Now consider the other term $M \kappa^{-2} G_{2 n}(\theta)$ in the probability bound (3.7). We choose $\kappa$ such that

$$
\kappa=\frac{(1-a) /(1-a) M^{-3 / 2}}{1+(1-a) /(1+a) M^{-3 / 2}} \geq \frac{1-a}{2(1+a)} M^{-3 / 2} .
$$

Hence,

$$
M \kappa^{-2} G_{2 n}(\theta) \leq\left(\frac{1-a}{2(1+a)}\right)^{2} M^{4} G_{2 n}(\theta) .
$$

Now we do not have the freedom anymore to choose $n$. We have to make the same choice as in (4.28). This yields

$$
M \kappa^{-2} G_{2 n}(\theta) \leq\left(\frac{2(1+a)}{(1-a)}\right)^{2} M^{4}\left(\frac{3 \beta^{3}}{8}\right)^{n(\theta)-1} .
$$

Requiring that the latter expression is less than $\epsilon / 2$ is equivalent to

$$
(n(\theta)-1) \ln \left(\frac{8}{3 \beta^{3}}\right) \geq \ln \left(8\left(\frac{1+a}{1-a}\right)^{2}\right)+4 \ln (M)+\ln \left(\epsilon^{-1}\right) .
$$

As in [14] a simple numerical test shows that a valid choice for $\beta=0.47$. This results in $a \leq 0.957$ and $n(\theta) \approx\lfloor 0.013 \theta\rfloor$. Hence, $\ln \left(8 /\left(3 \beta^{3}\right)\right) \approx 3.2459$ and $\ln \left(8((1+a) /(1-a))^{2}\right) \approx 9.7153$. Since $M \leq D$ there exists a constant $C_{2}$ (whose precise value may be calculated from the numbers above) such that $M \kappa^{-2} G_{2 n}(\theta) \leq \epsilon / 2$ provided

$$
N \geq C_{2} M\left(\ln (D)+\ln \left(\epsilon^{-1}\right)\right) .
$$

Choosing $C:=\max \left\{C_{1}, C_{2}\right\}$ completes the proof of Theorem 3.2.

\section{Numerical EXAMPLES}

To illustrate our theoretical results we present the image of Lena with $N^{2}=262144$ pixels $(512 \times$ 512 ) and its subsequent reconstruction with $15 \%, 30 \%, 50 \%$ and $75 \%$ of the total data information.

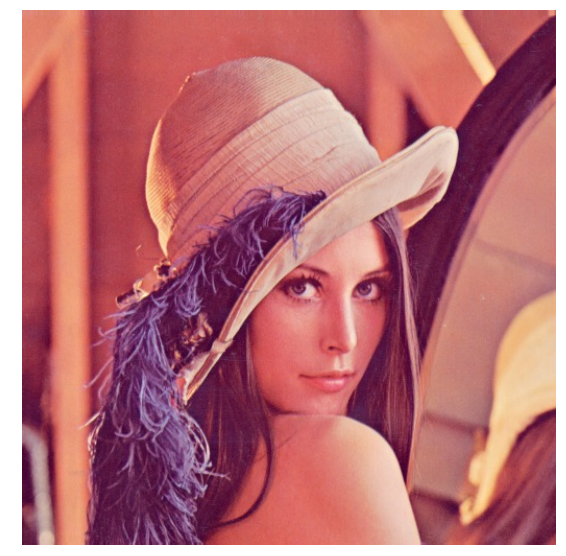

Figure 1. Original Lena image. 
The computations were made on a computer with Intel(R) Core(TM) i7-4790U CPU 3.60 GHz, RAM 16GB, Windows 8.1, OS 64-bit(win64) and running Matlab R2012b. Figure 1 corresponds to the original image.
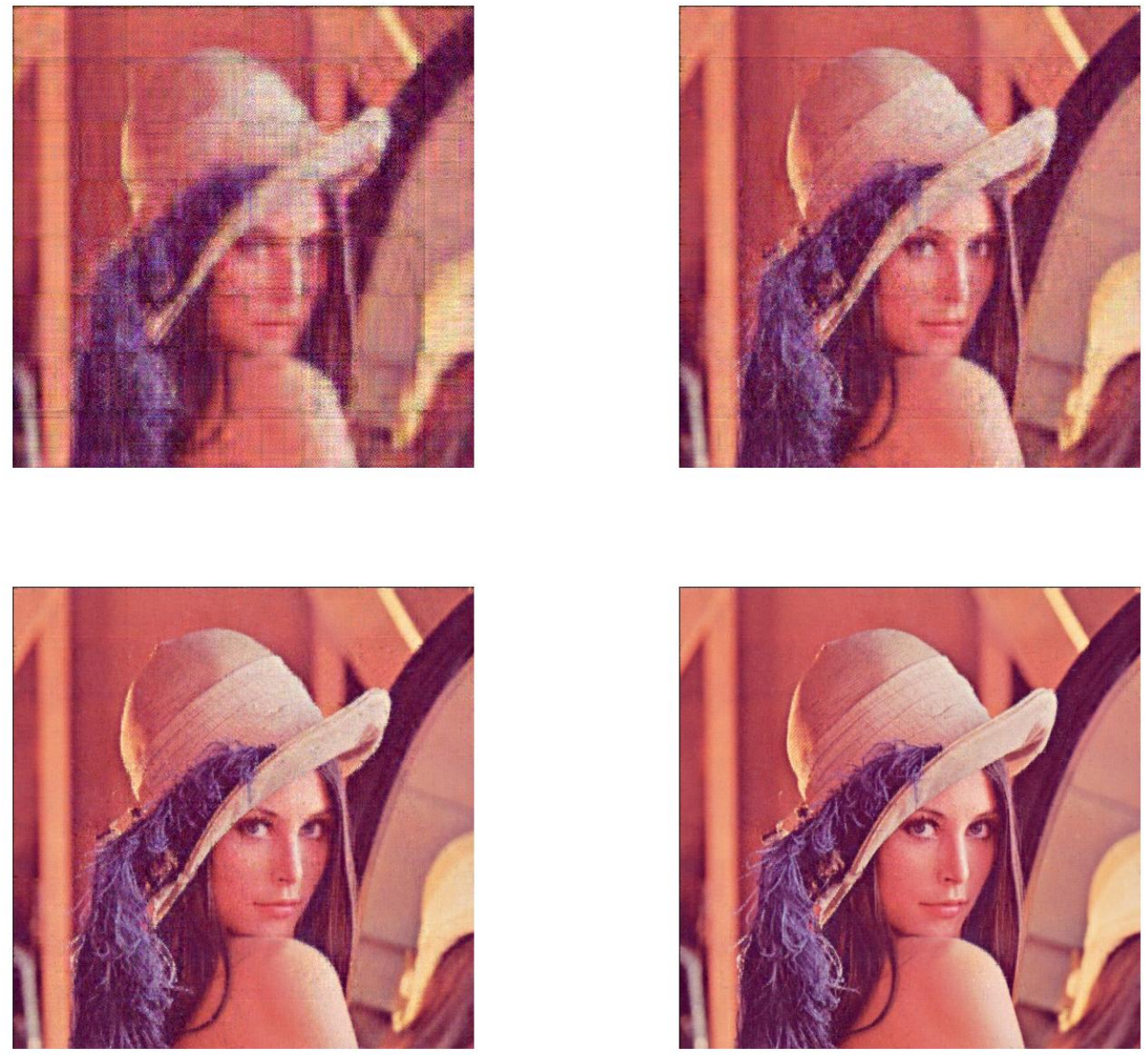

FIGURE 2 . Top left: reconstruction with $\approx 15 \%$ of the total information; top right: reconstruction with $\approx 30 \%$; bottom left: reconstruction with $\approx 50 \%$; bottom right: reconstruction with $\approx 75 \%$.

Figure 2 represents the different levels of reconstruction. Respectively, from left to right, top to bottom, the different sub-images correspond to reconstruction with approximately $15 \%, 30 \%, 50 \%$, and $75 \%$ of the total data information. The reconstructed images are blurred as expected but contain all relevant details. This shows that the concept of compressive sensing algorithms can be applied with a high probability of success in the bi-complex setting. 


\section{AcKnowledgements}

The work of the first and third authors was supported by Portuguese funds through the CIDMA

- Center for Research and Development in Mathematics and Applications, and the Portuguese Foundation for Science and Technology ("FCT-Fundação para a Ciência e a Tecnologia"), within project UID/MAT/ 0416/2013.

\section{REFERENCES}

[1] D. Alpay, et al. Basics of functional analysis with bicomplex scalars, and bicomplex Schur analysis, SpringerBriefs in Mathematics, Springer Cham Heidelberg New York Dordrecht London, 2014.

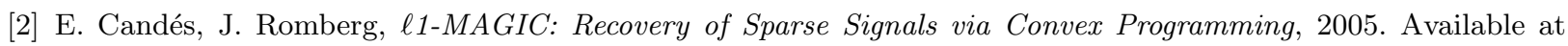
http://www.acm.caltech.edu/l1magic.

[3] E. Candés, J. Romberg, T. Tao, Robust uncertainty principles: Exact signal reconstruction from highly incomplete frequency information, IEEE Trans. Inform. Theory, 52 (2) (2006), 489-509.

[4] S. Chen, D. L. Donoho, Basis Pursuit: Technical report, Department of Statistics, Stanford University (1994), available at URL: http://statweb.stanford.edu/ donoho/Reports/1994/asilomar.pdf

[5] S. Chen, D. L. Donoho, and M. A. Saunders, Atomic decomposition by basis pursuit, SIAM J. Sci Comp., 20 (1) (1999), 33-61.

[6] D. L. Donoho, Compressed Sensing, IEEE Trans. Inform. Theory, 52 (4) (2006), 1289-1306.

[7] I. Gohberg, Continuous and Discrete Fourier Transforms, Extension Problems and Wiener-Hopf Equations, Vol. Operator Theory: Advances and Applications, Birkhüuser Basel, 2012.

[8] N. J. Higham, Functions of matrices: theory and computation. Siam, 2008.

[9] R. G. Lavoie, L. Marchildon and D. Rochon, Finite-Dimensional Bicomplex Hilbert Spaces, Adv. Appl. Clifford Algebr., 21 (3), (2011), 561-581.

[10] C. D. Meyer, Matrix Analysis and Applied Linear Algebra. SIAM, 2000.

[11] H. T. Nguyen, M. N. Do, Robust multichannel sampling, 15th Proc. IEEE Int. Conf. Image Process. 2008 , pp. 653-656.

[12] S. C. Pei, J.H. Chang, J.J. Ding, Commutative Reduced Biquaternions and Their Fourier Transform for Signal and Image Processing Applications, IEEE Trans. Signal Process., 52 (7) (2004), 2012-2031.

[13] S.C. Pei, J.J. Ding, J.H. Chang, Efficient implementation of quaternion Fourier transform, convolution, and correlation by 2-D complex FFT, IEEE Trans. Signal Process., 49 (11), (2001), 2783-2797.

[14] H. Rauhut, Random sampling of sparse trigonometric polynomials, Appl. Comput. Harmon. Anal. 22 (1) (2007), 16-42.

[15] G. Teschke, Multi-frame representations in linear inverse problems with mixed multiconstraints, Appl. Computat. Harmon. Anal. ,22 (1), (2007), 43-60

†CIDMA - Center for Research and Development in Mathematics and Applications, Department of Mathematics, University of Aveiro

Campus Universitário de Santiago

3810-193 Aveiro, Portugal

${ }^{\ddagger}$ FaCulty of Mathematics And Computer Science, Hubei University, Hubei, China

*Cidma - Center for Research and Development in Mathematics and Applications, Department of Science and Technology, University of Cape Verde,

Praia, CABo-Verde 